University of Nebraska - Lincoln

DigitalCommons@University of Nebraska - Lincoln

$8-1-2001$

\title{
Dynamic hyperpolarizability and two-photon detachment in the presence of a strong static electric field: Application to $\mathrm{H}^{-}$
}

M.V. Frolov

Voronezh State University, Voronezh, Russia

N.L. Manakov

Voronezh State University, manakov@phys.vsu.ru

Anthony F. Starace

University of Nebraska-Lincoln, astarace1@unl.edu

Follow this and additional works at: https://digitalcommons.unl.edu/physicsstarace

Part of the Physics Commons

Frolov, M.V.; Manakov, N.L.; and Starace, Anthony F., "Dynamic hyperpolarizability and two-photon detachment in the presence of a strong static electric field: Application to $\mathrm{H}^{-1 "}$ (2001). Anthony F. Starace Publications. 79.

https://digitalcommons.unl.edu/physicsstarace/79

This Article is brought to you for free and open access by the Research Papers in Physics and Astronomy at DigitalCommons@University of Nebraska - Lincoln. It has been accepted for inclusion in Anthony F. Starace Publications by an authorized administrator of DigitalCommons@University of Nebraska - Lincoln. 


\title{
Dynamic hyperpolarizability and two-photon detachment in the presence of a strong static electric field: Application to $\mathrm{H}^{-}$
}

\author{
M. V. Frolov and N. L. Manakov \\ Physics Department, Voronezh State University, 394693, Voronezh, Russia
}

Anthony F. Starace

Department of Physics and Astronomy, The University of Nebraska, Lincoln, Nebraska 68588-0111

(Received 30 November 2000; revised manuscript received 13 April 2001; published 13 July 2001)

\begin{abstract}
Our recent analysis [J. Phys. B 33, R141 (2000)] of the problem of $\mathrm{H}^{-}$in both laser and strong static electric fields, which treated effects that are linear in the laser intensity $I$ (e.g., the dynamic polarizability and the single-photon detachment cross section), is extended here to treat effects of higher order $\left(\sim I^{2}\right)$ in the laser intensity (e.g., the dynamic hyperpolarizability, the two-photon detachment cross section, the linear in $I$ corrections to the single-photon detachment rate, etc). We introduce the concept of the dynamic hyperpolarizability of an atom in the presence of a strong electric field based on the complex quasienergy approach with properly normalized, quasistationary quasienergy wave functions. Our analysis of the general structure of the dynamic hyperpolarizability tensor of an atom in a nondegenerate $(S$-) state is performed for arbitrary field geometries and laser polarizations. The connection of the hyperpolarizability $\gamma(\mathcal{F} ; \omega)$ to the complex quasienergy and to atomic ionization rates is established. Analytic results (in terms of Airy functions) are obtained for five irreducible components of a hyperpolarizability tensor (that are independent of the laser polarization and the field geometry) for the case of a weakly bound electron in a three-dimensional, zero-range potential. These results are used for the analysis of the frequency, field geometry, and laser polarization dependence of the two-photon detachment rate as well as of the linear in laser intensity corrections to the single-photon detachment rate. It is shown that the oscillatory behavior of the frequency dependence of both the real and the imaginary parts of $\gamma(\mathcal{F} ; \omega)$ exists for both the coplanar and orthogonal field geometries, and that this behavior is qualitatively different for frequencies below and above the single-photon detachment threshold. The threshold behavior of the two-photon detachment rate is analyzed in detail and the static electric-field-induced modification of Wigner's threshold law for a short-range potential is discussed.
\end{abstract}

DOI: 10.1103/PhysRevA.64.023417

PACS number(s): 32.80.Rm, 32.10.Dk, 32.80.Fb, 32.80.Gc

\section{INTRODUCTION}

Various methods have been discovered for controlling the outcome of particular laser-atom interactions. These methods include phase control in two-color experiments, polarization control by varying the polarization of the incident laser light, control by means of static fields, and control by combinations of these approaches. Particularly useful for controlling laser-atom processes are analytical theoretical results that predict the functional dependence of particular experimental observables on the control parameters. The point is that usually physical problems involve many parameters. For instance, for the problem considered here involving the joint action of laser and static fields, even for a fixed laser intensity there are five independent parameters: the frequency and polarization state of the laser beam, the static field strength, and the two angles describing the orientation of the static field with respect to the laser polarization ellipse. For such problems, time-consuming, completely numerical $a b$ initio calculations are typically performed only for limited sets of the control parameters, and hence, these relatively few results are only helpful as reference points for the generally less laborious analyses based on simple, analytically solvable, models that cover the entire parameter space. In laseratom physics, as in other fields, however, there are relatively few problems which permit analytic theoretical solutions.

One model system that does have analytical theoretical solutions for problems of interest in laser-atom physics is that of an electron bound in a short-range, $\delta$-function potential [1]. Although this model is applicable generally to represent only a short-range potential (e.g., as for a weakly bound electron in $\mathrm{H}^{-}$), the complex quasienergy solution for a $\delta$ potential in a strong monochromatic field [2] has been employed widely in laser-atom calculations and allows one to obtain a number of qualitative features that have been observed in real atoms, such as, e.g., the plateau structure in high-order harmonic generation [3]. In a recent review [4], we have employed the quasistationary, quasienergy approach to obtain the complex quasienergy for an electron bound in the three-dimensional, $\delta$-function potential in the presence of both a laser field and a strong static electric field. Generally, from the complex quasienergy, one is able to obtain the decay rate of an (initially bound) atomic system in both strong laser and static electric fields. In our review, we gave a complete account of the lowest order (linear) in laser intensity effects, which are entirely described in terms of the staticfield-dependent, (two-component) dynamic polarizability tensor. In particular, the photodetachment cross section is deduced from the imaginary part of the dynamic polarizability. In this paper we extend those results to the next order in the laser intensity, i.e., we treat the dynamic hyperpolarizability tensor (involving five independent components) in the presence of a strong static field and analyze the decay rates of second order in the laser intensity, which describe two- 
photon detachment as well as the linear in laser intensity corrections to the single-photon detachment rate.

The subject of laser-atom interactions in the presence of a static electric field has a long history, which we have briefly reviewed in Ref. [4]. We note here therefore only some works that have focused on multiphoton processes in the presence of a static electric field, and which are therefore of greatest relevance to the work presented here. Arutyunyan and Askar'yan [5] have given a qualitative overview of multiphoton detachment in the presence of a static electric field. Nikishov [6] has analyzed the special case of a lowfrequency, linearly polarized laser field. Slonim and Dalidchik [7] have analyzed the special case of a circularly polarized laser beam collinear with the static electric field. Manakov and Fainshtein [2] (see also [8]) have analyzed the static field ionization (tunneling) from a harmonic of the quasienergy state in a monochromatic laser field. Ostrovsky and Telnov [9] have performed a general analysis of multiphoton detachment for the case of a strong laser field and a weak static electric field. Gao and Starace [10] presented analytic formulas for $n$-photon detachment of an electron bound in a short-range potential in the presence of a strong static electric field in the limit of weak laser intensities; however, their approach ignores rescattering effects (i.e., the interaction of the escaping electron with the atomic core in both intermediate and final states). Bao et al. [11] presented results for the two-photon detachment cross section including the effect of rescattering processes. We note also completely numerical, nonperturbative calculations of laser detachment of $\mathrm{Li}^{-}$[12] and $\mathrm{H}^{-}$[13] in the presence of a static field that take into account electron correlation effects. These calculations show that many-electron effects do not change the qualitative predictions of previous single-electron analyses of the static-field-induced oscillatory structure and of the threshold behavior of photodetachment rates. Mercouris and Nicolaides [14] have performed also nonperturbative manyelectron calculations of multiphoton (including abovethreshold) detachment of $\mathrm{H}^{-}$in the presence of a static electric field; however, their numerical results are presented for only two laser frequencies and two values of the static field strength for fixed laser polarization, intensity, and field geometry.

In this paper we present in Sec. II a general analysis of the response of an atom to a laser field in the presence of a static electric field in the limit that the laser field amplitude is treated as a perturbation parameter. Our analysis treats all terms of second order in the laser intensity, which may be analyzed in terms of the hyperpolarizability tensor, similarly to the case of zero static field. Of course, in the presence of a static electric field, the general structure of the hyperpolarizability tensor is more involved. We use general symmetry and perturbation theory arguments to extract the dependence of the atomic response on the geometry of the fields and on the polarization of the laser and establish that the hyperpolarizability tensor of an atom for the case considered involves five independent (irreducible) components that are functions only of the laser frequency and the static field strength. Also in Sec. II we relate the "generalized" hyperpolarizability (composed of a set of these five irreducible components) to the complex quasienergy of the bound atomic state.

The results of Sec. II are very general and applicable to any atomic system in a nondegenerate (e.g., $S$ ) state. In Sec. III we carry the theoretical analysis further by considering the special case of an electron bound by a short-range, $\delta$-function potential. We present here analytic expressions for the two most important components of the hyperpolarizability tensor in terms of Airy functions and present numerical results for the generalized hyperpolarizability, which describes the Stark-shift and broadening (i.e., the decay rate) of a weakly bound level, taking into account the quadratic in laser intensity terms. To our knowledge there are no other results on static-field-dependent dynamic hyperpolarizabilities of negative ions with which we can compare in order to determine the accuracy of our zero-range potential model description of these complicated atomic parameters. However, for the case of zero static field, such results are available for $\mathrm{H}^{-}$both in a zero-range potential model [15] treatment and in nonperturbative [16] and perturbative (in the laser field) [17] many-electron numerical treatments employing correlated wave functions. In Sec. III B 1 we recover the result of Manakov et al. [15] as a special case of our more general results and we compare that with the numerical results of Nicolaides, Mercouris, and Piangos [16] and of Pipin and Bishop [17]. The comparison shows reasonable agreement (within about 10\%) with the most accurate manyelectron results in Ref. [17].

In Sec. IV we analyze in more detail the two-photon detachment cross section for the $\mathrm{H}^{-}$ion in the presence of a strong static field (i.e., the imaginary part of the generalized hyperpolarizability), presenting both analytical and numerical results. These results show that there are far more possibilities to control two-photon detachment by variation of the field parameters, the laser polarization, and the geometry of the two fields than are possible for the one-photon detachment process. For the energy region above the single-photon detachment threshold, we present our results for the total rate for laser detachment, which includes contributions from both the (above threshold) two-photon detachment process and the linear in laser intensity corrections to one-photon detachment. We know of no other works that have treated the latter corrections. Our results show that they can be negative, implying that they reduce the one-photon detachment rate. Finally, in Sec. V we present some conclusions.

\section{GENERAL FEATURES OF THE DYNAMIC HYPERPOLARIZABILITY TENSOR OF A BOUND $S$ STATE IN THE PRESENCE OF A STRONG STATIC ELECTRIC FIELD}

\section{A. Definitions and notation}

We consider the interaction of an atomic system with two fields, a static electric field, $\mathcal{F}$, and a monochromatic laser field having an arbitrary elliptic polarization,

$$
\mathbf{F}(t)=F \operatorname{Re}\{\mathbf{e} \exp [-i(\omega t-\mathbf{k} \cdot \mathbf{r})]\} .
$$

The length gauge is used for the dipole interaction Hamiltonian: 


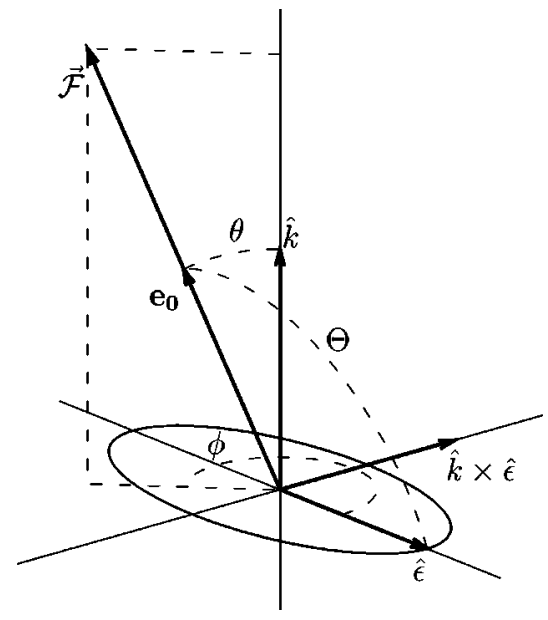

FIG. 1. Geometry for a static electric field, $\mathcal{F}=\mathcal{F} \mathbf{e}_{0}$, and a laser field, $\mathbf{F}(t)$, defined by Eqs. (1) and (3).

$$
V(\mathbf{r}, t)=|e|(\mathbf{F}(t)+\mathcal{F}) \mathbf{r}
$$

The most general geometry for the applied fields is considered, namely, that the direction of the vector $\mathcal{F}$ is defined by the polar angles $\theta, \phi$ in the coordinate frame whose $z$ axis is directed along the wave-vector $\mathbf{k}=\hat{k} \omega / c$ of the elliptically polarized laser field $\mathbf{F}(t)$ and whose $x$ axis is along the major semiaxis of the polarization ellipse, defined by the unit vector $\hat{\epsilon}$. The following parametrization of the unit complex polarization vector $\mathbf{e}\left(\mathbf{e} \cdot \mathbf{e}^{*}=1\right)$ is used for an elliptically polarized field $\mathbf{F}(t)$ with ellipticity parameter $\eta$ :

$$
\mathbf{e}=\frac{\hat{\epsilon}+i \eta \hat{k} \times \hat{\epsilon}}{\sqrt{1+\eta^{2}}}, \quad-1 \leqslant \eta \leqslant 1,
$$

where $\eta=0$ corresponds to linear polarization, and $\eta=$ $+1(-1)$ corresponds to right (left) circular polarization. These relationships are illustrated in Fig. 1 , where the unit vector $\mathbf{e}_{0}$ defines the direction of a static field, $\mathcal{F}=\mathcal{F} \mathbf{e}_{0}$, and where the angle $\Theta$ is the only one which enters the results for the case of a linearly polarized field $\mathbf{F}(t)$, where $\cos \Theta$ $=\sin \theta \cos \phi$. Instead of the ellipticity $\eta$, it is often convenient to employ the degrees of linear $(l)$ and circular $(\xi)$ polarization, which have invariant forms in terms of the vectors $\mathbf{e}$ and $\mathbf{e}^{*}$ :

$$
l=\frac{1-\eta^{2}}{1+\eta^{2}}=\mathbf{e} \cdot \mathbf{e}=\mathbf{e}^{*} \cdot \mathbf{e}^{*}, \quad \xi=\frac{2 \eta}{1+\eta^{2}}=i \hat{k} \cdot\left(\mathbf{e} \times \mathbf{e}^{*}\right) .
$$

As shown in [4], the dynamic polarizability as well as higher susceptibility tensors of a decaying system (whose decay, e.g., may be by means of field ionization in a strong static field and/or by means of laser-induced ionization) are determined by the Fourier component, $\tilde{\mathbf{d}}_{\omega}$, of the fieldinduced "dual" dipole moment

$$
\widetilde{\mathbf{d}}(t)=(1 / 2) \sum_{n=-\infty}^{\infty} \widetilde{\mathbf{d}}_{n \omega} \exp (-i n \omega t) .
$$

In contrast with the usual definition of the dipole moment $\mathbf{d}(t)$ of a stable system, this (dual) dipole moment $\widetilde{\mathbf{d}}(t)$ is generally complex, and its Fourier components $\widetilde{\mathbf{d}}_{\omega}$ and $\widetilde{\mathbf{d}}_{-\omega}$ are related by the condition

$$
\widetilde{\mathbf{d}}_{-\omega}=\left.\widetilde{\mathbf{d}}_{\omega}\right|_{\mathbf{e} \rightarrow \mathbf{e}^{*}} .
$$

In terms of the quasistationary, quasienergy state (QQES) wave function, $\Phi_{\epsilon}(\mathbf{r}, t)$, and its dual function, $\widetilde{\Phi}_{\epsilon}(\mathbf{r}, t)$, the definition of $\widetilde{\mathbf{d}}_{ \pm \omega}$ is [4]:

$$
\widetilde{\mathbf{d}}_{ \pm \omega}=\frac{2}{T} \int_{0}^{T} d t e^{ \pm i \omega t}\left\langle\widetilde{\Phi}_{\epsilon}(\mathbf{r}, t)|\hat{\mathbf{d}}| \Phi_{\epsilon}(\mathbf{r}, t)\right\rangle, \quad T=2 \pi / \omega
$$

where $\hat{\mathbf{d}}$ is the dipole moment operator [18]. These results are valid for an arbitrary $F$ (and for $\mathcal{F} \neq 0$ ). If we confine ourselves to the case of moderate or weak $F$, but assume that $\mathcal{F}$ may be strong, the following perturbative expansion in $F$ is valid:

$$
\widetilde{\mathbf{d}}_{\omega}=F \widetilde{\mathbf{d}}^{(1)}+F^{3} \widetilde{\mathbf{d}}^{(3)}+\cdots,
$$

where $\widetilde{\mathbf{d}}^{(n)}$ are $F$-independent vectors. The result in Eq. (7) follows simply from the perturbative expansion of $\Phi_{\epsilon}$ and $\widetilde{\Phi}_{\epsilon}$ in $F$ and from parity selection rules.

\section{B. Symmetry requirements}

Although explicit expressions for the vectors $\widetilde{\mathbf{d}}^{(n)}$ require explicit expressions for the QQES functions $\Phi_{\epsilon}$ and $\widetilde{\Phi}_{\epsilon}$, the geometrical and polarization dependence of these matrix elements can be deduced using only very general arguments based on symmetry considerations. Specifically, the vectors $\widetilde{\mathbf{d}}^{(n)}$ can be presented in terms of the vectors that appear in our problem (i.e., $\mathbf{e}, \mathbf{e}^{*}$ and $\mathbf{e}_{0}$ ) and the corresponding polarizabilities, which for $n>1$ are nonlinear polarizabilities (or susceptibilities). Since each vector $\widetilde{\mathbf{d}}^{(n)}$ (with $n=2 k+1$ ) is determined by the $n$th order of perturbation theory (PT) in $F$ [cf. Eq. (7)], its general form must comprise $(k+1)$ vectors $\mathbf{e}, k$ vectors $\mathbf{e}^{*}$, and hence, an even number of vectors $\mathbf{e}_{0}$. For example, since $\widetilde{\mathbf{d}}^{(1)}$ involves only the vectors $\mathbf{e}$ and $\mathbf{e}_{0}$, it must have the general form [4]:

$$
\widetilde{\mathbf{d}}^{(1)}=\alpha_{0}(\mathcal{F} ; \omega) \mathbf{e}+\alpha_{1}(\mathcal{F} ; \omega)\left(\mathbf{e} \cdot \mathbf{e}_{0}\right) \mathbf{e}_{0},
$$

where $\alpha_{0}$ and $\alpha_{1}$ are two geometry- and polarizationindependent atomic parameters, i.e., they are components of the dynamic polarizability tensor. For $n>1$, the analysis becomes more tedious, but is nevertheless straightforward. For the purposes of this paper, we only require the next vector, $\widetilde{\mathbf{d}}^{(3)}$.

The general form of $\widetilde{\mathbf{d}}^{(3)}$ involves 3 vector components,

$$
\tilde{\mathbf{d}}^{(3)}=\chi_{1} \mathbf{e}+\chi_{2} \mathbf{e}^{*}+\chi_{0} \mathbf{e}_{0},
$$


where the susceptibilities $\chi_{i}$ depend on the geometry of the fields and on the polarization state of the laser field. This dependence may be established using the explicit form of $\widetilde{\mathbf{d}}^{(3)}$ in terms of fourth order PT (in $F$ ) matrix elements. We obtain:

$$
\begin{gathered}
\chi_{1}=\gamma_{1}(\mathcal{F} ; \omega)+\gamma_{3}(\mathcal{F} ; \omega)\left|\mathbf{e} \cdot \mathbf{e}_{0}\right|^{2}, \\
\chi_{2}=l \gamma_{2}(\mathcal{F} ; \omega)+\gamma_{4}(\mathcal{F} ; \omega)\left(\mathbf{e} \cdot \mathbf{e}_{0}\right)^{2}, \\
\chi_{0}=\gamma_{3}(\mathcal{F} ; \omega)\left(\mathbf{e} \cdot \mathbf{e}_{0}\right)+l \gamma_{4}(\mathcal{F} ; \omega)\left(\mathbf{e}^{*} \cdot \mathbf{e}_{0}\right) \\
+4 \gamma_{5}(\mathcal{F} ; \omega)\left|\mathbf{e} \cdot \mathbf{e}_{0}\right|^{2}\left(\mathbf{e} \cdot \mathbf{e}_{0}\right) .
\end{gathered}
$$

In deriving Eq. (10) we have used the fact that $\mathbf{e} \cdot \mathbf{e}^{*}=1$ and the definition $\mathbf{e} \cdot \mathbf{e}=l$. We note also the nonself-evident fact that the parameters $\gamma_{3}$ and $\gamma_{4}$ in $\chi_{0}$ are the same as those in $\chi_{1}$ and $\chi_{2}$. These identities may be established using explicit PT expressions for the $\chi_{i}$ 's.

In general, as shown in Eq. (10), the hyperpolarizability tensor in the presence of a static field involves five independent ("irreducible") components $\gamma_{i}, 1 \leqslant i \leqslant 5$, for an arbitrary field geometry and an arbitrary elliptic polarization of $\mathbf{F}(t)$. For various special cases, however, fewer independent parameters suffice. For the case $\mathcal{F}=0$, only two of them, $\gamma_{1}$ and $\gamma_{2}$, are nonzero and they determine the hyperpolarizability tensor for a nondegenerate atomic level in a monochromatic light field (see, e.g., [19]). Moreover, for $\mathcal{F}=0$ and particular cases of laser polarization only one parameter contributes: for the case of a circularly polarized laser field, this is $\gamma_{1}$; for the case of linear polarization, it is $\gamma_{1}+\gamma_{2}$. For $\mathcal{F} \neq 0$ and for linear polarization of $\mathbf{F}(t)$, three parameters contribute: $\gamma_{1}+\gamma_{2}, \gamma_{3}+\gamma_{4}$, and $\gamma_{5}$. For weak $\mathcal{F}$, the dependence of $\gamma_{i}$ on $\mathcal{F}$ is as follows:

$$
\gamma_{3}(\mathcal{F} ; \omega) \sim \mathcal{F}^{2}, \quad \gamma_{4}(\mathcal{F} ; \omega) \sim \mathcal{F}^{2}, \quad \gamma_{5}(\mathcal{F} ; \omega) \sim \mathcal{F}^{4}
$$

\section{Relation to the complex quasienergy}

As for the case of $\mathcal{F}=0$, the hyperpolarizability tensor for the case $\mathcal{F} \neq 0$ describes a number of nonlinear optical effects that occur when laser radiation passes through an atomic medium in the presence of a static field (such as, e.g., the intensity-dependent correction to the refractive index). Furthermore, it determines the linear in intensity correction to the cross section for (Rayleigh) light scattering. In particular, this correction may be important for analyses of polarization anomalies induced by a static field, such as staticelectric-field-induced circular dichroism in nonresonant light scattering by atoms [4]. Moreover, a detailed analysis shows that this correction leads to new dichroic effects that occur only for the case of elliptic polarization of $\mathbf{F}(t)$ (termed "elliptic dichroism") and that vanish for the case of completely circular polarization.

Besides its importance for nonlinear optical applications, the dual dipole moment is related closely to the spectral characteristics of an atom in static and laser fields. Namely, the derivative in $F$ of the (complex) quasienergy $\epsilon$ of an atom when both fields are strong is connected with $\widetilde{\mathbf{d}}_{\omega}$ as follows [4]:

$$
\frac{\partial \epsilon}{\partial F}=-\frac{1}{2} \mathbf{e}^{*} \cdot \tilde{\mathbf{d}}_{\omega} .
$$

Obviously, due to the static field $\mathcal{F}$ an initial unperturbed atomic state $\left|\psi_{0}\right\rangle$ with the energy $E_{0}$ is transformed into a quasistationary state with the wave-function $\Phi_{E}(\mathbf{r})$ and a complex energy $E$. Using the expansion (7) and Eq. (12), the linear and squared in laser intensity corrections to the energy $E$ may be expressed in terms of the parameters $\alpha_{i}(\mathcal{F} ; \omega)$ and $\gamma_{i}(\mathcal{F} ; \omega)$. Using Eqs. (3) and (4) and the chosen geometry (see Fig. 1), we obtain for the (complex) scalar product e. $\mathbf{e}_{0}$ :

$$
\mathbf{e} \cdot \mathbf{e}_{0}=\frac{\sin \theta}{\sqrt{2(1+l)}}[(1+l) \cos \phi+i \xi \sin \phi]=\left|\mathbf{e} \cdot \mathbf{e}_{0}\right| e^{i \phi_{1}},
$$

where

$$
\begin{gathered}
\left|\mathbf{e} \cdot \mathbf{e}_{0}\right|=\Lambda / \sqrt{2}, \quad \Lambda=\sin \theta \sqrt{1+l \cos 2 \phi}, \\
\tan \phi_{1} \equiv \tan \left[\arg \left(\mathbf{e} \cdot \mathbf{e}_{0}\right)\right]=\eta \tan \phi .
\end{gathered}
$$

Using these notations and Eqs. (8)-(10), (12), the expansion of the quasienergy $\epsilon$ in $F$ up to terms $\sim F^{4}$ may be written as

$$
\Delta \epsilon \equiv \epsilon-E=-\frac{1}{4} \alpha(\mathcal{F} ; \omega) F^{2}-\frac{1}{24} \gamma(\mathcal{F} ; \omega) F^{4},
$$

where

$$
\alpha(\mathcal{F} ; \omega)=\alpha_{0}(\mathcal{F} ; \omega)+\frac{1}{2} \Lambda^{2} \alpha_{1}(\mathcal{F} ; \omega),
$$

and

$$
\gamma(\mathcal{F} ; \omega)=3\left[\gamma_{1}+l^{2} \gamma_{2}+\Lambda^{2} \gamma_{3}+l \Lambda^{2} \cos 2 \phi_{1} \gamma_{4}+\Lambda^{4} \gamma_{5}\right]
$$

are the dynamic polarizability and the hyperpolarizability respectively.

The imaginary part of $\epsilon, \Gamma=-2 \operatorname{Im} \epsilon / \hbar$, gives the total decay rate of an initial bound state in both static and laser fields. For not too high $\mathcal{F}$ (so that $\operatorname{Im} E$ remains small) and for frequencies $\omega$ comparable with $\left|E_{0}\right| / \hbar$, the laser-fieldinduced ionization is the only important process. Specifically, for $\hbar \omega>\left|E_{0}\right|, \operatorname{Im} \alpha(\mathcal{F} ; \omega)$ determines the one-photon ionization rate and $\operatorname{Im} \gamma(\mathcal{F} ; \omega)$ gives the linear in laser intensity correction to the one-photon ionization rate as well as the direct (above threshold) two-photon detachment rate. For $\left|E_{0}\right|>\hbar \omega>\left|E_{0}\right| / 2, \operatorname{Im} \alpha(\mathcal{F} ; \omega)$ is exponentially small and $\operatorname{Im} \gamma(\mathcal{F} ; \omega)$ determines the rate of two-photon ionization in the presence of a static field. The explicit expressions for the 
$\gamma_{i}$ 's and for $\gamma(\mathcal{F} ; \omega)$ in terms of the matrix elements of fourth order PT for the QQES have the same formal structure as that for $\mathcal{F}=0$ (cf. [21]): the difference is that the atomic Green functions should be replaced by those that include the presence of a static field (denoted here by $\mathcal{G}_{E \pm k \hbar \omega}\left(\mathbf{r}, \mathbf{r}^{\prime}\right)$, with $k=0,1$, and 2) and also the properly normalized (dual) bra-vector, $\left\langle\widetilde{\Phi}_{E}(\mathbf{r})\right|$, should be used instead of $\left\langle\psi_{E_{0}}(\mathbf{r})\right|$. However, the evaluation of such matrix elements for real atoms is, of course, a formidable task. In the rest of this paper we present the analysis of $\gamma(\mathcal{F} ; \omega)$ for a short-range potential, in which case the final results may be obtained in closed analytical form.

\section{STATIC-FIELD-DEPENDENT DYNAMIC HYPERPOLARIZABILITY FOR THE ZERO-RANGE POTENTIAL MODEL}

\section{A. Definitions and (scaled) units}

We have obtained analytically the irreducible components of the hyperpolarizability tensor, $\gamma_{i}(\mathcal{F} ; \omega)$ in Eq. (10), and the combined hyperpolarizability, $\gamma(\mathcal{F} ; \omega)$ in Eq. (17), for an electron described by the bound state wave function,

$$
\psi_{0}(\mathbf{r})=N \frac{e^{-\kappa r}}{r}, \quad N=\sqrt{\kappa / 2 \pi},
$$

for a zero-range ( $\delta$-model) potential,

$$
U(r)=\frac{2 \pi \hbar^{2}}{k m} \delta(\mathbf{r}) \frac{\partial}{\partial r} r
$$

with the binding energy $E_{0}=-\hbar^{2} \kappa^{2} / 2 m$. In order to present our results in the most general form, we use the following scaled units (sc.u): energies and frequencies are measured in units of $\left|E_{0}\right|$ and $\left|E_{0}\right| / \hbar$, the length unit is $1 / \kappa$; and the laser field amplitude $F$ and the static field amplitude $\mathcal{F}$ are measured in units of the "internal field," $F_{0}=\sqrt{2 m\left|E_{0}\right|^{3}} /|e| \hbar$. The virtue of using such scaled units is that our results apply to systems having different binding energies, $\left|E_{0}\right|$, but for different values of the field strengths. As an example, for $\mathrm{H}^{-}$ we have $F_{0}^{H^{-}}=3.362 \times 10^{7} \mathrm{~V} / \mathrm{cm}$, and the corresponding scaled unit of the intensity, $I=c F^{2} / 8 \pi$, is $I_{0}^{H^{-}}=c F_{0}^{2} / 8 \pi$ $=1.498 \times 10^{12} \mathrm{~W} / \mathrm{cm}^{2}$. Thus, in scaled units, we have $I$ $=F^{2}$. The cross section $\sigma$ in our units $\left(\kappa^{-2}\right)$ is connected with that in atomic units, $\sigma^{\text {(a.u.) }}$, by the relation: $\sigma^{\text {(a.u.) }}$ $=\sigma\left(E_{a} / 2\left|E_{0}\right|\right)$, where $E_{a}=m e^{4} / \hbar^{2}=1$ a.u. Finally, in order to apply our results to the special case of $\mathrm{H}^{-}$, our results for (hyper)polarizabilities and detachment cross sections should be multiplied by the "renormalization" factor $A_{c}=2.6551$ that is based on effective range theory and that is more appropriate for comparison with experiment than is the usual normalization of the ground-state wave function in Eq. (18) for the $\delta$-function potential [20].

\section{B. Expressions for $\gamma_{1}(\mathcal{F} ; \omega)$ and $\gamma_{2}(\mathcal{F} ; \omega)$ in terms of Airy functions and zero-static-field limit}

Our analysis is based on the use of the quasistationary quasienergy state (QQES) approach [21], which is similar to the well-known quasistationary (or resonance) state approach for radiationless atomic problems for time-independent Hamiltonians. A detailed analysis of the QQES problem for our case of a combination of the $\delta$-model potential and two (generally strong) external fields has been presented in a recent review [4], where the analytical techniques for the perturbative account of a laser field have been developed for the case of $\alpha_{0}(\mathcal{F} ; \omega)$ and $\alpha_{1}(\mathcal{F} ; \omega)$ [cf. Eq. (8)]. Using similar techniques, we performed for our present purposes two independent calculations for $\gamma(\mathcal{F} ; \omega)$ : one based on the use of a perturbative expansion (up to terms of order $F^{3}$ ) of the exact result for $\widetilde{\mathbf{d}}_{\omega}$ [cf. Eq. (6)], and one starting from the perturbative result for $\widetilde{\mathbf{d}}^{(3)}$ [cf. Eqs. (9), (10)] in terms of the Green's function, $\mathcal{G}_{\mathcal{E}}\left(\mathbf{r}, \mathbf{r}^{\prime}\right)$, of an electron in the zero-range potential and in a static electric field. Both methods provided the same results for $\gamma_{i}(\mathcal{F} ; \omega)$ in terms of regular $\operatorname{Ai}(\xi)$ and irregular $\operatorname{Bi}(\xi)$ Airy functions [22] and their derivatives.

Since the general expressions for $\gamma_{i}$ involve 3 Green functions $\mathcal{G}_{E \pm k \omega}\left(\mathbf{r}, \mathbf{r}^{\prime}\right)$ (with different $k$ ), the final results are quite lengthy. Thus, we present here only those for $\gamma_{1}(\mathcal{F} ; \omega)$ and $\gamma_{2}(\mathcal{F} ; \omega)$, which are the only nonzero ones for $\mathcal{F}=0$. Also, only these parameters contribute to $\gamma(\mathcal{F} ; \omega)$ for the case of orthogonal geometry, $\Lambda=0$ [cf. Eq. (14)]. The analytical expressions for $\gamma_{i}(\mathcal{F} ; \omega), 3 \leqslant i \leqslant 5$, have been presented elsewhere [23]. For the simplest presentation of results it is convenient to introduce the following combinations of Airy functions $\operatorname{Ai}(\xi)$ and $\operatorname{Ci}(\xi)=\operatorname{Bi}(\xi)+i \operatorname{Ai}(\xi)$, and their derivatives:

$$
J(\xi)=\operatorname{Ai}^{\prime}(\xi) \operatorname{Ci}^{\prime}(\xi)-\xi \operatorname{Ai}(\xi) \operatorname{Ci}(\xi),
$$

$$
I(\xi)=-J^{\prime}(\xi)=\operatorname{Ai}(\xi) \operatorname{Ci}(\xi)
$$

Note that $J(\xi)$ is the regularized part (at $\mathbf{r}=\mathbf{r}^{\prime} \rightarrow 0$ ) of the Green's function for a free electron in a static electric field [4]. Next we define auxiliary functions $Q\left(\xi_{n}\right)$ and $K\left(\xi_{n}\right)$ :

$$
\begin{gathered}
Q\left(\xi_{n}\right)=\xi_{n}^{2} J\left(\xi_{n}\right)+\frac{1}{4} \xi_{n} I^{\prime}\left(\xi_{n}\right)+\frac{3}{8} I\left(\xi_{n}\right), \\
K\left(\xi_{n}\right)=\xi_{n} J\left(\xi_{n}\right)+\frac{1}{4} I^{\prime}\left(\xi_{n}\right),
\end{gathered}
$$

where

$$
\xi_{n}=(n \omega-E) \mathcal{F}^{-2 / 3}, \quad n=0, \pm 1, \pm 2 .
$$

In terms of the functions introduced above, the result for $\gamma_{1}(\mathcal{F} ; \omega)$ may be written as: 


$$
\begin{aligned}
\gamma_{1}(\mathcal{F}, \omega)= & \frac{8 \pi \mathcal{F}^{5 / 3}}{15 \omega^{8} L(\mathcal{F})}[R(\mathcal{F}, \omega)+R(\mathcal{F},-\omega) \\
& \left.-\frac{5}{3} \mathcal{F}^{2 / 3} \frac{\partial}{\partial E} \frac{\left[K\left(\xi_{-1}\right)-2 K\left(\xi_{0}\right)+K\left(\xi_{1}\right)\right]^{2}}{I\left(\xi_{0}\right)}\right],
\end{aligned}
$$

where

$$
R(\mathcal{F}, \omega)=Q\left(\xi_{2}\right)-4 Q\left(\xi_{1}\right)+3 Q\left(\xi_{0}\right)
$$

and where

$$
L(\mathcal{F})=2 \pi \mathcal{F}^{-1 / 3} I\left(-E \mathcal{F}^{-2 / 3}\right)
$$

is the (complex) normalization factor for the quasistationary state $\Phi_{E}(\mathbf{r})$ in a static field [4].

The explicit result for $\gamma_{2}(\mathcal{F}, \omega)$ may be presented in a form similar to Eq. (20):

$$
\begin{aligned}
\gamma_{2}(\mathcal{F}, \omega)= & \frac{4 \pi \mathcal{F}^{5 / 3}}{15 \omega^{8} L(\mathcal{F})}[R(\mathcal{F}, \omega)+R(\mathcal{F},-\omega)-S(\mathcal{F}, \omega) \\
& -S(\mathcal{F},-\omega)]
\end{aligned}
$$

where

$$
S(\mathcal{F}, \omega)=\frac{5 \pi \mathcal{F}^{1 / 3}}{3} \frac{\left[K\left(\xi_{0}\right)-2 K\left(\xi_{1}\right)+K\left(\xi_{2}\right)\right]^{2}}{1+\pi \mathcal{F}^{1 / 3} J\left(\xi_{2}\right)} .
$$

We note that the parameter $E$ in the above equations is the exact (complex) energy of the quasistationary state $\Phi_{E}(\mathbf{r})$ in a static field. The dependence of $E$ on $\mathcal{F}$ has been analyzed in detail in Ref. [4], where it was shown that for not too strong $\mathcal{F}(\mathcal{F}<0.01)$ and far from threshold frequencies the approximation $E \simeq E_{0}=-1$ may be used. The other components, $\gamma_{i}(\mathcal{F} ; \omega)$ with $i=3,4,5$, have structures that are similar to (though more cumbersome than) those of Eqs. (20) and (22). Thus, except for the prefactor $1 / \omega^{8}$ in $\gamma_{i}(\mathcal{F} ; \omega)$, the frequency $\omega$ enters the results only in the arguments of the Airy functions, defined by Eq. (19). Obviously, these arguments with $n=0$ correspond to effects of a static field only, while the terms with $n=-1$ and $n=-2$ (or $n=1$ and $n=2$ ) correspond to the absorption (or stimulated emission) of one and two photons, respectively.

\section{The zero-static-field limit}

As mentioned above [see Eq. (11)], for $\mathcal{F} \rightarrow 0$ the dominant contributions to $\gamma(\mathcal{F} ; \omega)$ are given by $\gamma_{1}(\mathcal{F} ; \omega)$ and $\gamma_{2}(\mathcal{F} ; \omega)$, which are the only nonzero components of the hyperpolarizability tensor at $\mathcal{F}=0$. Using the known asymptotic expansions [4] of the functions $J(\xi)$ and $I(\xi)$ at $\xi \rightarrow \pm \infty$ (i.e., $\mathcal{F} \rightarrow 0$ ), we obtain the results for $\gamma_{1,2}(\mathcal{F}$ $=0 ; \omega) \equiv \gamma_{1,2}(\omega)$, which coincide with those first obtained in Ref. [15] by direct perturbative calculations of the quasienergy in the QQES approach:
TABLE I. The static polarizability, $\alpha$, and the static hyperpolarizability, $\gamma_{z z z z}$, of $\mathrm{H}^{-}$(in a.u.)

\begin{tabular}{lccc}
\hline \hline & Ref. [17] & Ref. [16] & Present work $^{\mathrm{a}}$ \\
\hline$\alpha$ & 206.165 & 201.8 & 215.53 \\
$\gamma_{z z z z} \mathrm{~b}$ & $8.03 \times 10^{7}$ & $7.6 \times 10^{7}$ & $9.08 \times 10^{7}$ \\
\hline
\end{tabular}

"The "renormalization" factor $A_{c}$ for $\mathrm{H}^{-}$was taken into account in these results.

${ }^{\mathrm{b}}$ In terms of $\gamma_{1}$ and $\gamma_{2}, \gamma_{z z z z}=8\left(\gamma_{1}+\gamma_{2}\right)$.

$$
\begin{aligned}
& \gamma_{1}(\omega)=\frac{8}{45 \omega^{8}}\left[f_{1}(\omega)+f_{1}(-\omega)\right], \\
& \gamma_{2}(\omega)=\frac{8}{45 \omega^{8}}\left[f_{2}(\omega)+f_{2}(-\omega)\right],
\end{aligned}
$$

where

$$
\begin{aligned}
f_{1}(\omega)=45 \omega^{2}+96+5 i\left(\omega^{2}-1\right)^{1 / 2}\left(\omega^{2}-7\right) & \\
- & 4 i(\omega-1)^{1 / 2}\left(3 \omega^{2}+14 \omega-32\right)+3 i(2 \omega-1)^{5 / 2}, \\
f_{2}(\omega)= & 15 \omega^{2}+28-\frac{1}{\omega}\left[10(\omega-1)^{5 / 2}(2 \omega-1)^{1 / 2}\right. \\
& +i(2 \omega-1)^{1 / 2}\left(9 \omega^{3}+24 \omega^{2}-26 \omega+10\right) \\
& \left.-2 i(\omega-1)^{1 / 2}\left(7 \omega^{3}-14 \omega^{2}+12 \omega-5\right)\right] .
\end{aligned}
$$

Note that we define $\sqrt{-a}$ as $i \sqrt{a}$ for $a>0$. This convention corresponds to an exponentially decreasing wave in closed detachment channels and to a spherically diverging wave in open channels.

The analytical structure of the results in Eqs. (23), (24) is consistent with well-known square-root peculiarities of cross sections for a short-range potential [24], which are reflections of the threshold behaviors of hyperpolarizabilities at the opening of two- $(\omega \geqslant 1 / 2)$ and one-photon $(\omega \geqslant 1)$ detachment channels (see Ref. [15] for details). Despite the simplicity of the model, the analytical results for a zerorange potential in Eqs. (23) and (24) [as well as those for the polarizability $\alpha(\omega)[25,15]]$ are in reasonable agreement with sophisticated numerical calculations of hyperpolarizabilities for $\mathrm{H}^{-}$employing electron-correlated wave functions [16,17]. Specifically, in Ref. [17], the nonlinear optics definitions for two independent components of the hyperpolarizability tensor, $\gamma_{z z z z}(-\omega ; \omega, \omega,-\omega)$ and $\gamma_{x x z z}(-\omega$; $\omega, \omega,-\omega)$, are used [for $\omega=0, \gamma_{x x z z}=(1 / 3) \gamma_{z z z z}$ ]. In terms of $\gamma_{1}(\omega)$ and $\gamma_{2}(\omega)$ we have $\gamma_{z z z z}=8\left(\gamma_{1}+\gamma_{2}\right), \quad \gamma_{x x z z}$ $=4 \gamma_{1}$. Taking the limits of Eqs. (23), (24) at $\omega=0$, we obtain the zero-range potential model result for $\mathrm{H}^{-}: \gamma_{z z z z}$ $=\frac{9}{4} A_{c}$ (sc.u.) $=9.08 \times 10^{7}$ (a.u.). In Table I, we compare the results for the static polarizability and the hyperpolarizability $\gamma_{z z z z}$ of $\mathrm{H}^{-}$in the zero-range potential model with those in Refs. [16,17]. One sees that the results of Pipin and Bishop [17] (which are the most accurate to our knowledge) lie be- 


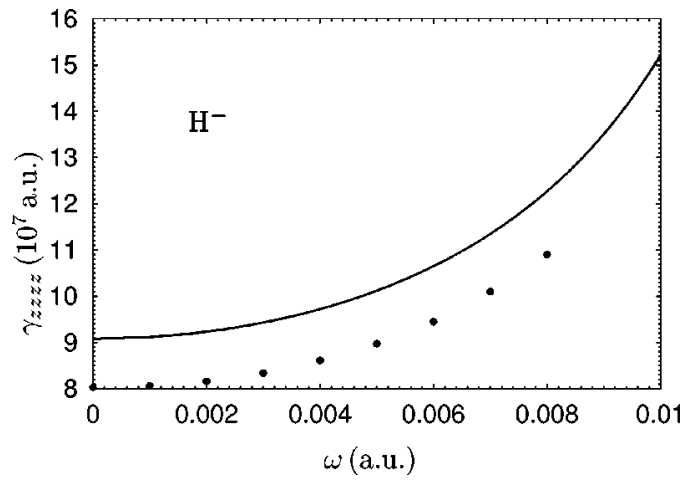

FIG. 2. Frequency dependence of the component $\gamma_{z z z z}(-\omega$; $\omega, \omega,-\omega)$ of the dynamic hyperpolarizability tensor of $\mathrm{H}^{-}$. Solid line: Zero-range potential model result [Eqs. (23),(24)]. Solid points: The results of Pipin and Bishop [17].

tween the results of Refs. [16] and [15]. Figure 2 presents a comparison of predictions for the frequency-dependent hyperpolarizabilities. It is seen that the accuracy is uniform over the frequency interval considered. The $10 \%$ accuracy of the zero-range potential model for predictions of such complicated atomic parameters as hyperpolarizabilities seems acceptable to us given the ability of the model to provide analytically the dependence of $\gamma(\mathcal{F} ; \omega)$ on $\omega, \mathcal{F}$, the laser polarization, and the field geometry. A $10 \%$ accuracy also seems acceptable given the existing level of accuracy of intense laser experiments.

A further indication that our zero-range potential model results are quite reasonable is provided by the more detailed comparison with the nonperturbative, many-electron numerical results of Nicolaides, Mercouris, and Piangos [16] shown in Fig. 3. Figure 3(a) compares predictions for $\alpha(\omega)$ for 0 $\leqslant \omega \leqslant 0.25$ a.u. and Fig. 3(b) compares predictions for $\gamma(\omega)$ for $0 \leqslant \omega \leqslant 0.08$ a.u. One sees that except near the thresholds, our predictions and those of Ref. [16] agree very well.

\section{Numerical results for real and imaginary parts of $\gamma(\mathcal{F} ; \omega)$}

The dependence of $\gamma(\mathcal{F} ; \omega)$ on the polarization and the geometry is described by the factors $l, \cos 2 \phi_{1}$, and $\Lambda^{2}$ $=\sin ^{2} \theta(1+l \cos 2 \phi)$ in Eq. (17). This analytic equation shows that for an arbitrary geometry $(\Lambda \neq 0)$ both real and imaginary parts of $\gamma(\mathcal{F} ; \omega)$ are sensitive to the polarization state of the laser field. Moreover, in contrast to results for the dynamic polarizability [4], the polarization dependence of $\gamma(\mathcal{F} ; \omega)$ is significant even for the case of an orthogonal geometry. In Figs. 4 and 5, we present numerical results for the real and imaginary parts, respectively, of the hyperpolarizability $\gamma(\mathcal{F} ; \omega)$ for three values of the static field $\mathcal{F}$ for an orthogonal field geometry for either a linearly or a circularly polarized laser field. Note that for the case of circular polarization with the laser beam collinear with the static field (i.e., $\hat{k} \| \mathbf{e}_{0}$ or $\left.\Lambda=0\right), \operatorname{Re} \gamma(\mathcal{F} ; \omega)=3 \operatorname{Re} \gamma_{1}$; and for the case of a linearly polarized laser beam collinear with the static field (i.e., also $\Lambda=0), \operatorname{Re} \gamma(\mathcal{F} ; \omega)=3 \operatorname{Re}\left(\gamma_{1}+\gamma_{2}\right)$ [cf. Eq. (17)].

Note that $\operatorname{Im} \gamma(\mathcal{F} ; \omega)$ is related to the contribution of or$\operatorname{der} F^{4}$ to the total decay rate $\Gamma$, i.e., $\Gamma^{(4)}=(\operatorname{Im} \gamma / 12) F^{4}$, where
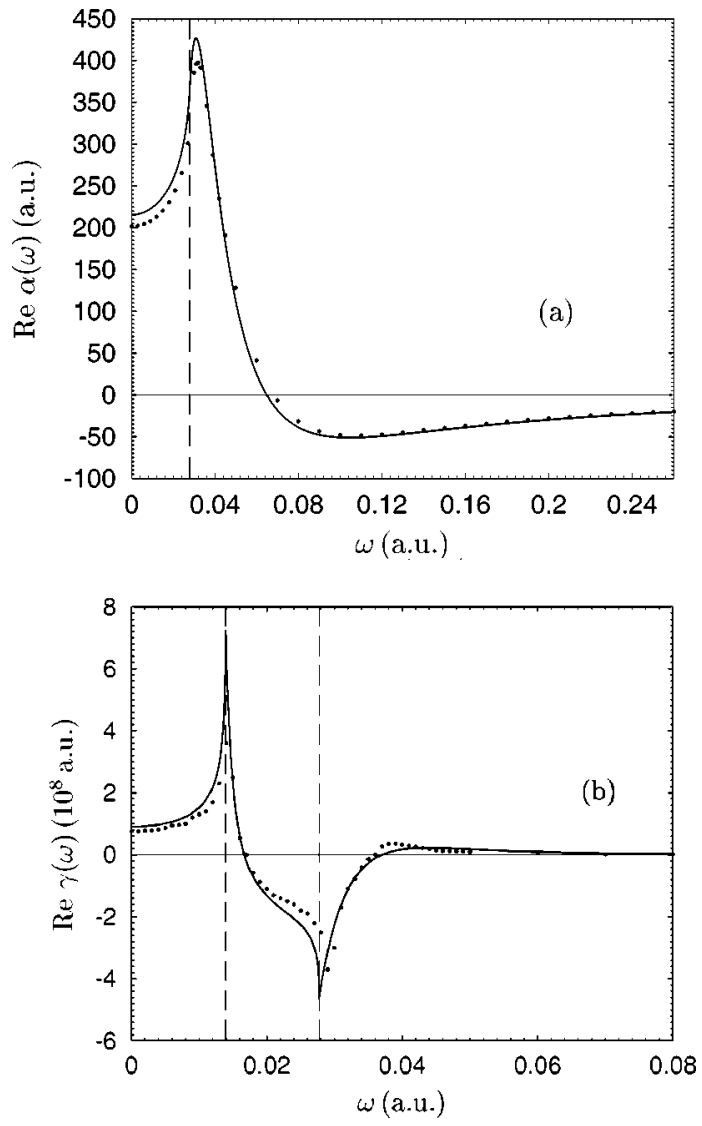

FIG. 3. Comparison of zero-range potential model and nonperturbative many-electron numerical predictions for $\mathrm{H}^{-}$. (a) Frequency dependence of the real part of the dynamic polarizability $\alpha(\omega)$ for $0 \leqslant \omega \leqslant 0.25$ a.u. (b) Frequency dependence of the real part of the hyperpolarizability $\gamma(\omega)$ for $0 \leqslant \omega \leqslant 0.08$ a.u. Solid lines: Present zero-range potential model results. Solid circles: Nonperturbative, many-electron numerical results of Ref. [16].

$$
\Gamma=\Gamma_{\text {stat. }}+\Gamma^{(2)}+\Gamma^{(4)},
$$

and where $\Gamma^{(2)} \sim F^{2}$ and $\Gamma_{\text {stat. }}=-2 \operatorname{Im} E$ is the static-fieldinduced decay rate. As Figs. 4 and 5 demonstrate, the oscillatory behavior of the hyperpolarizability $\gamma$ as a function of $\omega$ (in the field configuration in which the laser polarization vector and the static field direction are orthogonal) contrasts with the behavior of the dynamic polarizability, which does not oscillate [4]. For the case of a linearly polarized laser field and orthogonal geometry, the oscillation pattern is more pronounced for $\omega<1$ than for $\omega>1$ (where $\omega=1$ is the single photon ionization threshold for $\mathcal{F}=0$ ). For the case of circular polarization, however, oscillations appear only for $\omega>1$. Another general result that is clear from Figs. 4 and 5 is that increasing the static field leads (on average) to a reduction of both the real and the imaginary parts of $\gamma(\mathcal{F} ; \omega)$.

Our numerical results for the case of coplanar or collinear geometry are presented in Figs. 6 and 7 for both the case that $\mathcal{F}$ is in the plane of circular polarization of $\mathbf{F}(t)$ (i.e., $\Lambda$ $=1, l=0)$ and the case that $\mathcal{F}$ is parallel to the direction of linear polarization of $\mathbf{F}(t)$ (i.e., $\Lambda=\sqrt{2}$ ). Both real and imaginary parts of $\gamma(\mathcal{F} ; \omega)$ exhibit oscillations about the re- 

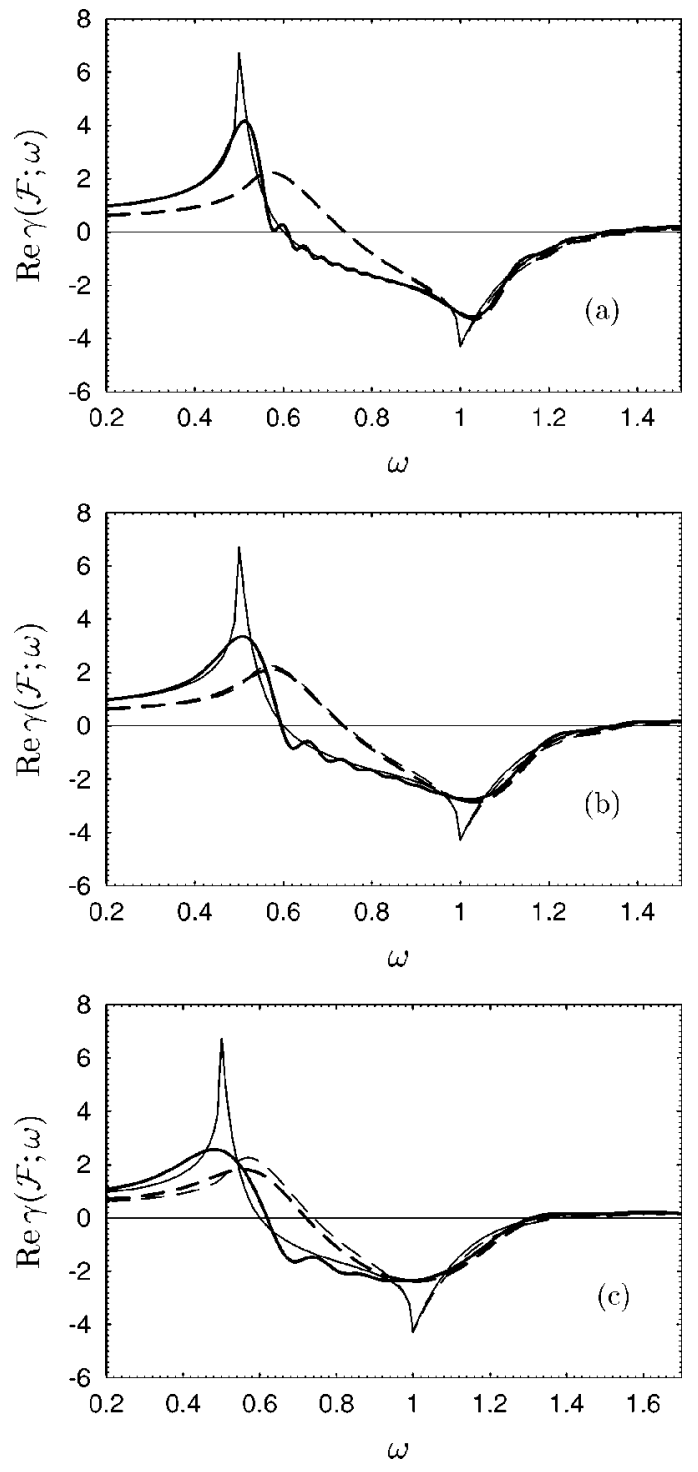

FIG. 4. Frequency dependence of the real part of the hyperpolarizability $\gamma(\mathcal{F} ; \omega)$ for the orthogonal field geometry for three values of the static field: (a) $\mathcal{F}=0.015$; (b) $\mathcal{F}=0.03$; and (c) $\mathcal{F}=0.06$. Solid line: linear laser polarization; $\operatorname{Re} \gamma(\mathcal{F} ; \omega)=3 \operatorname{Re}\left(\gamma_{1}+\gamma_{2}\right)$. Dashed line: circular laser polarization; $\operatorname{Re} \gamma(\mathcal{F} ; \omega)=3 \operatorname{Re} \gamma_{1}$. Thin solid line: Result for $\mathcal{F}=0$ and linearly polarized $\mathbf{F}(t)$. Thin dashed line: Result for $\mathcal{F}=0$ and circularly polarized $\mathbf{F}(t)$. Scaled units are used; see text for explanation.

sult for $\mathcal{F}=0$, which is given by Eqs. (23) and (24). These oscillations have the same (interference) origin as for the dynamic polarizability and they vanish for $\omega \gg 1$, where static field effects are negligible. For the coplanar or collinear geometry (i.e., when the static field is in the plane of circular polarization or parallel to the direction of linear polarization), the oscillation pattern is more pronounced than for an orthogonal field geometry. Moreover, in contrast with the case of orthogonal fields, oscillations are more pronounced for $\omega \gtrsim 1$ than for the case of two-photon ionization, $1 / 2<\omega<1$. Thus, the oscillation pattern is smoothed in the multiphoton case (for decreasing $\omega<1$ ). The static field ef-
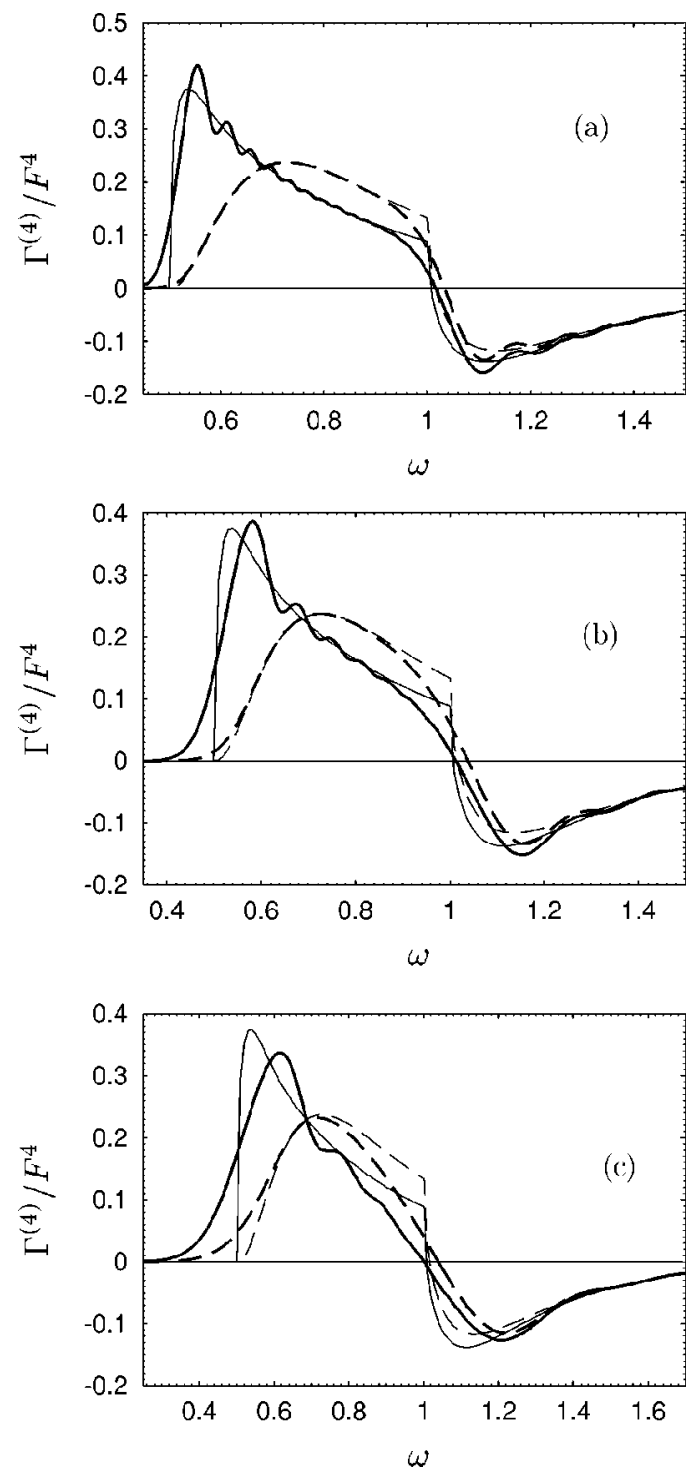

FIG. 5. The same as Fig. 4, but for (one twelfth) the imaginary part of the hyperpolarizability, $\operatorname{Im} \gamma(\mathcal{F} ; \omega)=12 \Gamma^{(4)} / F^{4}$.

fects are most important for near-threshold frequencies, $\omega$ $\simeq 1,1 / 2,1 / 3, \ldots$, and they smooth the square-root threshold peculiarities of cross sections that are typical for short-range potentials. As a result, for small frequencies (even for weak static fields), it is impossible to present the total decay rate $\Gamma$ in terms of partial rates with a fixed number of absorbed photons.

\section{TWO-PHOTON DETACHMENT OF $\mathrm{H}^{-}$ IN THE PRESENCE OF A STATIC FIELD}

\section{A. Results in alternative approximations, laser polarizations, and field geometries for $\omega<1$}

In this section, we analyze in more detail the imaginary part of $\gamma(\mathcal{F} ; \omega)$, which describes not only the two-photon detachment rate but also the linear in laser intensity corrections to the one-photon detachment rate. First, we consider 

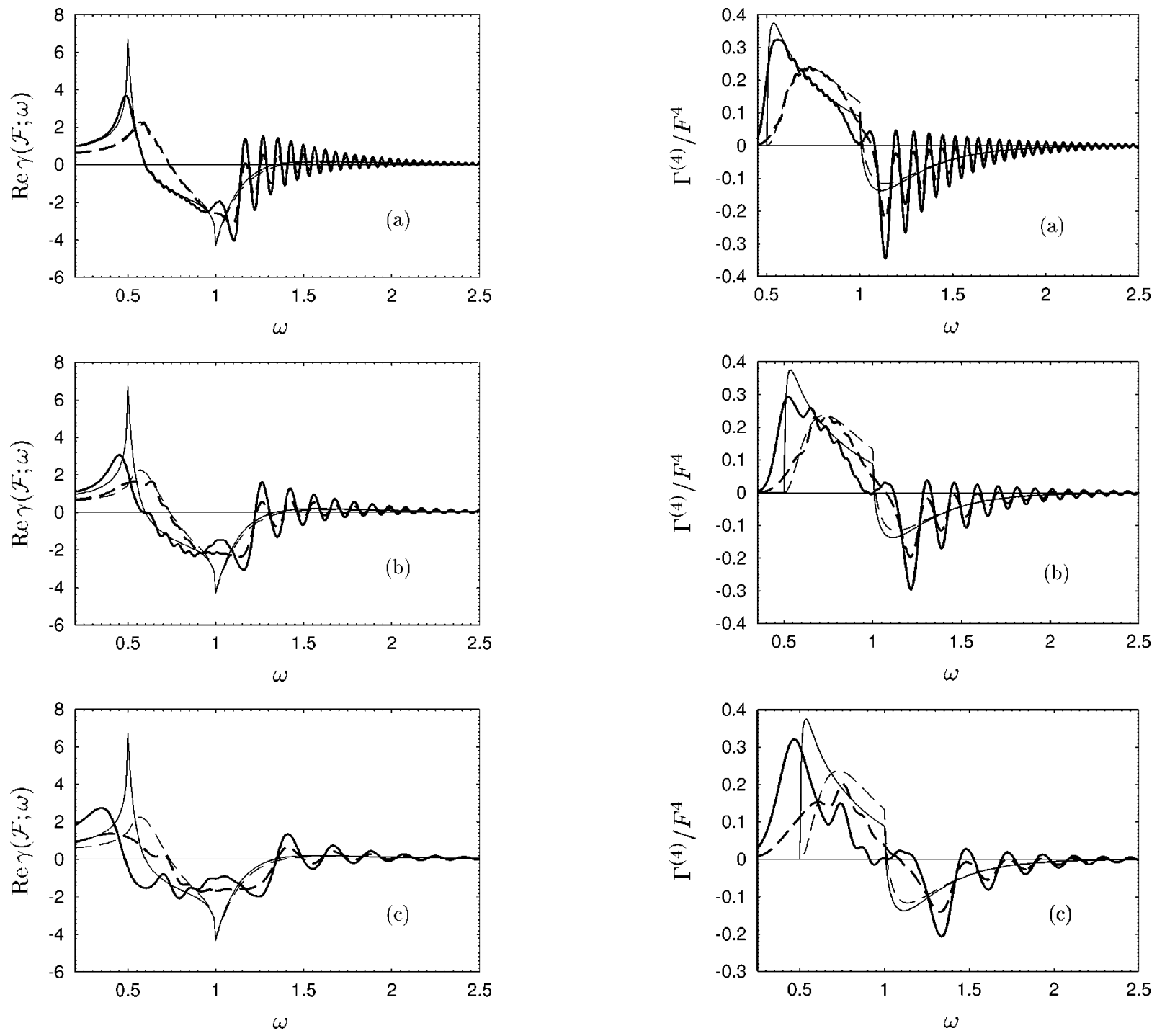

FIG. 6. Frequency dependence of the real part of the hyperpolarizability $\gamma(\mathcal{F} ; \omega)$ for coplanar or collinear geometry for three values of $\mathcal{F}$ : (a) $\mathcal{F}=0.015$; (b) $\mathcal{F}=0.03$; and (c) $\mathcal{F}=0.06$. Solid line: $\mathcal{F}$ collinear with the direction of linear polarization of $\mathbf{F}(t)(l$ $\left.=1, \Lambda^{2}=2\right)$. Dashed line: $\mathcal{F}$ in the plane of circular polarization of $\mathbf{F}(t) \quad\left(l=0, \Lambda^{2}=1\right)$. Thin solid line: Result for $\mathcal{F}=0$ and linearly polarized $\mathbf{F}(t)$. Thin dashed line: Result for $\mathcal{F}=0$ and circularly polarized $\mathbf{F}(t)$. Scaled units are used.

the case of two-photon detachment of $\mathrm{H}^{-}$for frequencies below the single-photon threshold, $1 / 2 \lesssim \omega \lesssim 1$. (Note that we use the symbol $\lesssim$ instead of $\leqslant$ since in the presence of a static field the threshold frequencies are not well defined.) For the frequency interval considered, the term $\Gamma^{(2)}$ in Eq. (25) is negligibly small (see Ref. [4], where the one-photon detachment rate for $\mathrm{H}^{-}$in the presence of a strong static field has been analyzed in detail). Thus, the laser-field-induced decay rate is proportional to $F^{4}=I^{2}$, and its dependence on polarization and frequency is presented in Figs. 5 and 7 for a number of values of $\mathcal{F}$. For moderate $\mathcal{F}$, it is possible to extract the dominant (in $\mathcal{F}$ ) terms from our exact analytical results for $\gamma_{i}(\mathcal{F} ; \omega)$. To compare these results with those of

FIG. 7. The same as Fig. 6, but for (one twelfth) the imaginary part of $\gamma, \operatorname{Im} \gamma(\mathcal{F} ; \omega)=12 \Gamma^{(4)} / F^{4}$.

other authors it is convenient to use the $F$-independent "generalized cross section:"

$$
\hat{\sigma}^{(2)}=\left[\frac{8 \pi \omega \alpha}{F^{2}}\right]^{2} \Gamma^{(4)}=\frac{16}{3}(\pi \alpha \omega)^{2} \operatorname{Im} \gamma(\mathcal{F} ; \omega) .
$$

Note that $\hat{\sigma}^{(2)}$ in Eq. (26) is expressed in scaled units; to convert it to ordinary units, multiply by the factor $1 / 4\left(E_{a} /\left|E_{0}\right|\right)^{3} a^{4} \hbar / E_{a}$, where $E_{a}$ is the atomic unit of energy and $a$ is the Bohr radius. For $\mathrm{H}^{-}$, for which $\left|E_{0}\right|$ $=0.027751$ a.u., this factor is $2.2188 \times 10^{-46} \mathrm{~cm}^{4} \mathrm{~s}$. Obviously, with the use of the exact result for $\operatorname{Im} \gamma(\mathcal{F} ; \omega), \hat{\sigma}^{(2)}$ in Eq. (26) gives not only the cross section for direct twophoton detachment, but describes also the contributions $\sim F^{4}$ to the rates for static field ionization and stimulated twophoton emission as well as the linear in laser intensity corrections to the rates for one-photon absorption and emission. However, for the considered interval of frequencies and for 
$\mathcal{F} \leq 0.05$, the contributions of all these channels are exponentially small and may be neglected.

\section{Alternative approximations and comparison with exact results. The case of collinear geometry}

As for the analysis of one-photon detachment of $\mathrm{H}^{-}$in Ref. [4], one may analyze different levels of approximation for taking account of static field effects in calculations of the two-photon detachment amplitude, $A_{f i}^{(2)}$, for negative ions:

$$
A_{f i}^{(2)}=\left\langle\psi_{f}\left|\mathbf{e} \cdot \mathbf{r} \mathcal{G}_{E+\omega}\left(\mathbf{r}, \mathbf{r}^{\prime}\right) \mathbf{e} \cdot \mathbf{r}^{\prime}\right| \Phi_{E}\right\rangle
$$

The simplest approximation (denoted $I$ ) consists in neglecting static-field effects in the (static-field-dressed) initial-state $\Phi_{E}(\mathbf{r})$ and in using results for the intermediate-state Green function $\mathcal{G}_{E+\omega}$ and for the final-state $\psi_{f}$ of the detached electron that are appropriate for a free electron in a static field. (This approximation I may be called a static-field Born approximation.) It is not possible to perform an exact analytical calculation of the amplitude in Eq. (27) in approximation $I$. The situation is similar to that for single-photon detachment, which has been analyzed in Ref. [4]. Specifically, for singlephoton detachment the transition amplitude in approximation $I$ also cannot be evaluated analytically. However, it can be evaluated using a saddle-point approximation, in which case it reduces exactly to the result of assuming a weak static field and performing an asymptotic expansion for the Airy functions in the exact analytic result for $\operatorname{Im} \alpha(\mathcal{F} ; \omega)$. Reasoning by analogy, we simply define approximation $I$ for twophoton detachment by the weak field, approximate expression of our analytic result for $\operatorname{Im} \gamma(\mathcal{F} ; \omega)$. Specifically, for weak fields, asymptotic expansions can be used for the Airy functions whose arguments are large and positive, namely $\xi_{0}, \xi_{ \pm 1}$, and $\xi_{2}$. Taking into account only Airy functions with the negative argument $\xi_{-2}=-(E+2 \omega) / \mathcal{F}^{2 / 3} \approx(1$ $-2 \omega) / \mathcal{F}^{2 / 3}$, and neglecting the imaginary part of Airy functions with other arguments and also the $\mathcal{F}$ dependence of the normalization factor in Eq. (21), we obtain after some algebra the following result in approximation $I$ (for the case of a linearly polarized laser field collinear with $\mathcal{F}$ ):

$$
\begin{aligned}
\hat{\sigma}_{I}^{(2)}= & A_{c} \frac{4 \pi^{3} \alpha^{2} \mathcal{F}^{1 / 3}}{15 \omega^{6}}\left[\left(\mathrm{Ai}^{\prime 2}-\xi \mathrm{Ai}^{2}\right)\left(127 \omega^{2}-152 \omega+48\right)\right. \\
& \left.-8 \mathcal{F}^{2 / 3}(12-19 \omega) \mathrm{Ai} \mathrm{Ai}^{\prime}-12 \mathcal{F}^{4 / 3} \mathrm{Ai}^{2}\right]
\end{aligned}
$$

where $\xi \equiv \xi_{-2}, \quad \operatorname{Ai} \equiv \operatorname{Ai}\left(\xi_{-2}\right), \quad \operatorname{Ai}^{\prime} \equiv \operatorname{Ai}^{\prime}\left(\xi_{-2}\right)$, and $A_{c}$ $=2.6551$ is a "renormalization" factor for $\mathrm{H}^{-}$.

In the weak static field limit, the oscillatory structure of cross sections is very simple for "far from threshold" frequencies,

$$
(2 \omega-1) \gg \mathcal{F}^{2 / 3},
$$

when the asymptotics of Airy functions for large negative arguments $\xi_{-2}$ may be used. In particular, for this case, the result in Eq. (28) reduces to elementary functions :

$$
\begin{aligned}
\lim _{\phi_{2} \gg 1} \hat{\sigma}_{I}^{(2)} \simeq & A_{c} \frac{(2 \pi \alpha)^{2}}{15 \omega^{6}} \sqrt{2 \omega-1}\left[127 \omega^{2}-152 \omega+48\right. \\
& \left.-5\left(49 \omega^{2}-56 \omega+16\right) \frac{\cos \phi_{2}}{\phi_{2}}\right]
\end{aligned}
$$

where

$$
\phi_{2}=\frac{4}{3} \frac{(2 \omega-1)^{3 / 2}}{\mathcal{F}}=\frac{4}{3}\left(-\xi_{-2}\right)^{3 / 2} .
$$

Thus, the parameter $\phi_{2}$ determines both the period and the amplitude of the oscillatory part of the cross section. Note that in the weak field limit the two-photon cross section depends on $\mathcal{F}$ only through the factor $\left(\cos \phi_{2}\right) / \phi_{2}$, which is the same dependence found for the one-photon detachment cross section $[7,20]$.

A better approximation (denoted $I I$ ) involves, in addition to the interactions included in approximation $I$, the exact account of the static field distortion of the initial state (18), by means of using the quasistationary wave-function $\Phi_{E}$. For this case the amplitude in Eq. (27) may be calculated analytically and the result for $\hat{\sigma}^{(2)}$ in the approximation $I I$ is:

$$
\begin{aligned}
\hat{\sigma}_{I I}^{(2)}= & A_{c} \frac{64 \pi^{3} \alpha^{2} \mathcal{F}^{1 / 3}}{\omega^{4}}\left[\mathrm{Ai}^{\prime 2}\left(\frac{1}{16}-\frac{3 \mathcal{F}^{2}}{2 \omega^{3}}+\frac{\mathcal{F}^{4}}{\omega^{6}}\right)\right. \\
& -\xi \mathrm{Ai}^{2}\left(\frac{1}{4}+\frac{\mathcal{F}^{2}}{\omega^{3}}\right)^{2}-\xi^{2}\left(\mathrm{Ai}^{2}-\mathrm{Ai}^{\prime 2}\right) \frac{\mathcal{F}^{2 / 3}}{6 \omega}\left(1-\frac{4 \mathcal{F}^{2}}{\omega^{3}}\right) \\
& -\mathrm{Ai} \mathrm{Ai}^{\prime} \frac{\mathcal{F}^{2 / 3}}{6 \omega}\left(1+\frac{20 \mathcal{F}^{2}}{\omega^{3}}\right)-\mathrm{Ai}^{2} \frac{3 \mathcal{F}^{4 / 3}}{10 \omega^{2}}\left(1+\frac{20 \mathcal{F}^{2}}{3 \omega^{3}}\right) \\
& \left.-\xi\left(\xi^{2} \mathrm{Ai}^{2}-\xi \mathrm{Ai}^{\prime 2}+2 \mathrm{Ai} \mathrm{Ai}^{\prime}\right) \frac{\mathcal{F}^{4 / 3}}{5 \omega}\right]
\end{aligned}
$$

where the notations are the same as for Eq. (28). Moreover, this result coincides exactly with that obtained by Gao and Starace [10] [after one performs an analytical calculation of the integral in their Eq. (53) for the case $N=2]$. Thus, as was pointed out for the case of one-photon detachment [4], for the perturbative account of a laser field, the method suggested in Ref. [10] is more accurate than the "static-field Born approximation" and is equivalent to an exact account of initial-state effects that are ignored in approximation $I$. We note that, as in the case of approximation II for one-photon detachment, it is impossible to extract the result (32) from our exact results for $\operatorname{Im} \gamma(\mathcal{F} ; \omega)$ by taking into account the corrections of higher orders in $\mathcal{F}$ compared to those accounted for in approximation $I$. The reason is that these high order corrections overlap with similar corrections caused by rescattering effects. These effects originate from the interaction of the detached electron with the binding potential (both in intermediate states, i.e., after absorption of one photon, and in the continuum). These rescattering effects are neglected in both approximations $I$ and $I I$. For two-photon detachment, the account of rescattering effects beyond approxi- 

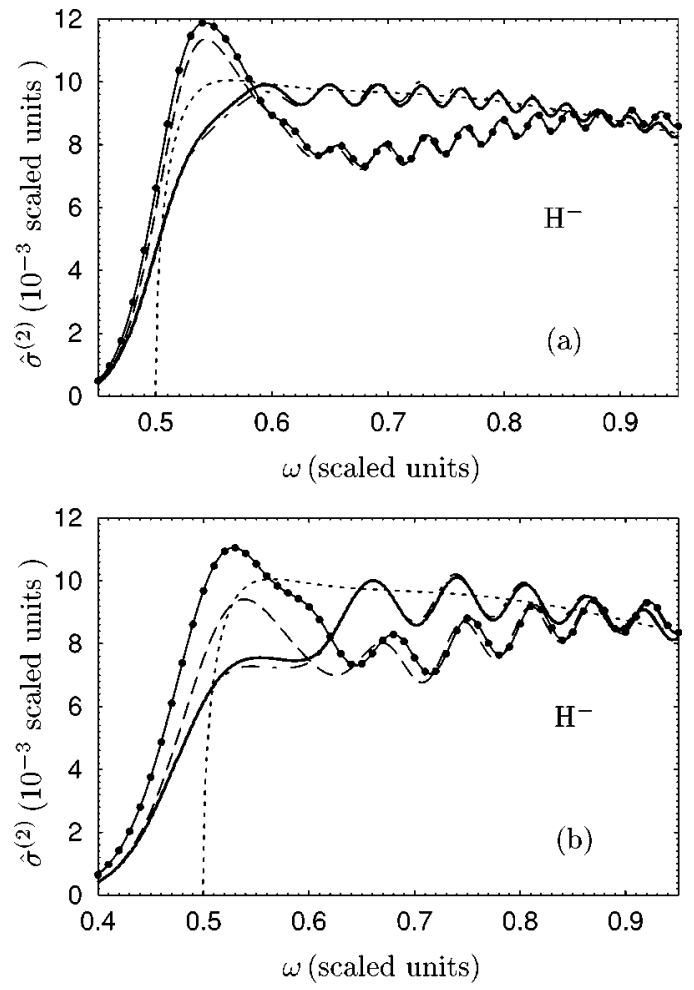

FIG. 8. Frequency dependence of $\hat{\sigma}^{(2)}$ for linear laser polarization $(l=1)$ and for collinear static field geometry for (a) $\mathcal{F}$ $=0.015$ and (b) $\mathcal{F}=0.03$. Solid line: exact result, Eqs. (17) and (26). Solid circles: approximation I, Eq. (28). Dashed line: approximation II, Eq. (32) (Ref. [10]). Dot-dashed line: approximation III (Ref. [11]). Dotted line: $\mathcal{F}=0$ result, Eqs. (17), (23), and (26) (Ref. [17]). For $\mathrm{H}^{-}$, the scaled units for $\hat{\sigma}^{(2)}$ and $\hbar \omega$ are 2.2188 $\times 10^{-46} \mathrm{~cm}^{4} \mathrm{~s}$ and 0.027751 a.u., respectively.

mation $I$ (i.e., using the unperturbed initial state) was performed in Ref. [11] together with account of initial-state effects as in approximation $I I$. Thus, in this approximation (denoted III) only the interference terms are neglected, which can only be taken into account by a simultaneous treatment of both rescattering and initial state effects.

The comparison of results calculated in approximations $I$, $I I$, and $I I I$ with our exact calculations, employing Eqs. (17) and (26), is presented in Fig. 8. One observes good agreement of the results in approximation $I I I$ with our exact results except for a narrow interval of near-threshold frequencies. [Note that the arguments of Ref. [26] concerning the (possible) inaccuracy of the results in Ref. [11] is thus shown here for the two-photon case to be incorrect. A detailed discussion of these assertions for the one-photon case has been given elsewhere [27].]

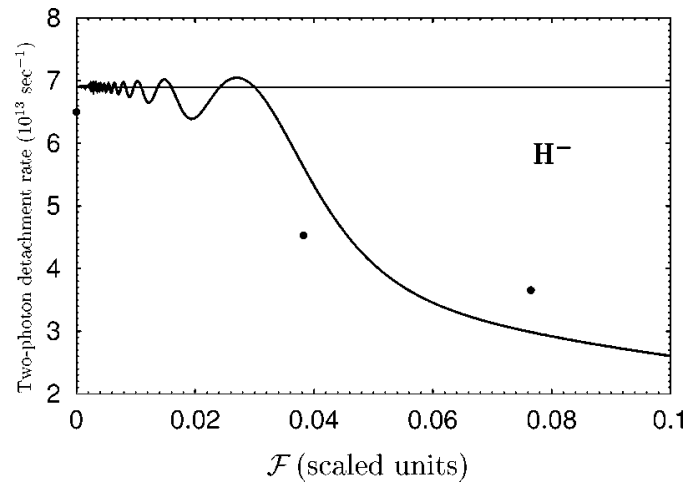

FIG. 9. $\mathcal{F}$ dependence of the two-photon detachment rate of $\mathrm{H}^{-}$ for the case of a linearly polarized $(l=1)$ laser field and a collinear field geometry $(\Lambda=\sqrt{2})$ at $\omega=0.6487$ and $F=0.305976$ (in scaled units). Thick solid line: present result. Thin solid line: the zerorange result for $\mathcal{F}=0$. Solid points: Results of Mercouris and Nicolaides [14].

In order to verify the accuracy of the zero-range potential model for the description of multiphoton detachment of $\mathrm{H}^{-}$ in combined laser and static fields, in Table II we compare our results for the two-photon detachment rate $\Gamma^{(4)}$ [calculated using Eq. (26)] with the nonperturbative, correlatedelectron calculations [14] for the collinear geometry and the following set of field parameters: $\omega=0.018$ a.u. $=0.65 \mathrm{sc} . \mathrm{u}$.; $F=2.0 \times 10^{-3}$ a.u. $=0.31$ sc.u.; and for three values of $\mathcal{F}: 0$, $2.5 \times 10^{-4}$ a.u. $=0.03825$ sc.u., $\quad$ and $5.0 \times 10^{-4}$ a.u. $=0.0765 \mathrm{sc} . \mathrm{u}$. Since for the laser intensity considered, $I$ $=F^{2}=0.096$, the use of a perturbative approach is questionable, we also calculated $\Gamma^{(4)}$ for $\mathcal{F}=0$ nonperturbatively, based on the exact equations for the complex quasienergy for the zero-range potential model. This result is $\Gamma^{(4)}=0.5$ $\times 10^{13} \mathrm{sec}^{-1}$. Thus we expect that higher order in laser intensity corrections cannot change qualitatively our results presented in Table II. In Fig. 9 we present the $\mathcal{F}$ dependence of the two-photon detachment rate for the frequency $\omega$ $=0.65$. While the results in Ref. [14] are presented for only few values of $\mathcal{F}$, we believe that the zero-range potential model and the numerical many-electron results are in reasonable agreement.

As a further indication that our zero-range potential model predictions are reliable, we present in Fig. 10 predictions for the ionization rate for $\mathrm{H}^{-}$above the two-photon ionization threshold. In order to compare with the results in Fig. 5 of Ref. [13], we have performed calculations for the same field ratios $\mu=\mathcal{F} / F$ employed in that paper. Comparison of our results in Fig. 10 with those in Fig. 5 of Ref. [13] shows excellent qualitative agreement below $\omega=0.03$ a.u. For $\omega$ $>0.03$ a.u. our predictions show somewhat more oscillatory

TABLE II. Two-photon detachment rates $\Gamma^{(4)}\left(\right.$ in $\left.\mathrm{sec}^{-1}\right)$ of $\mathrm{H}^{-}$for collinear linearly polarized laser and static electric fields: $\omega=0.65$ sc.u. ( $=0.018$ a.u. $), F=0.31$ sc.u $\left(=2 \times 10^{-3}\right.$ a.u. $)$.

\begin{tabular}{lccc}
\hline \hline & $\mathcal{F}=0$ & $\mathcal{F}=0.03825 \mathrm{sc} . \mathrm{u}=2.5 \times 10^{-4}$ a.u. & $\mathcal{F}=0.0765$ sc.u. $=5 \times 10^{-4}$ a.u. \\
\hline Ref. [14] & $0.65 \times 10^{13}$ & $0.45 \times 10^{13}$ & $0.36 \times 10^{13}$ \\
Present work & $0.69 \times 10^{13}$ & $0.56 \times 10^{13}$ & $0.3 \times 10^{13}$ \\
\hline \hline
\end{tabular}




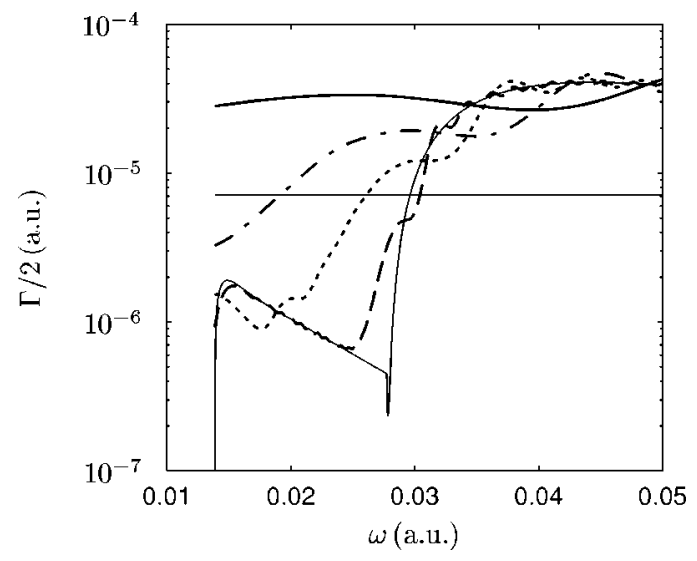

FIG. 10. Detachment rate $\Gamma / 2$ (a.u.) for $\mathrm{H}^{-}$as a function of frequency $\omega$ (a.u.) above the two-photon detachment threshold for various values of the static field $\mathcal{F}$. The laser intensity is $I=1.75$ $\times 10^{10} \mathrm{~W} / \mathrm{cm}^{2}$, which is equivalent to $F=0.10823$ scaled units. The value of $\mathcal{F}$ is indicated by the ratio $\mu=\mathcal{F} / F$. Solid thin line: $\mu=0$. Dashed line: $\mu=0.106$. Dotted line: $\mu=0.424$. Dot-dashed line: $\mu=1$. Solid thick line: $\mu=2$. These results should be compared with those in Fig. 5 of Ref. [13].

structure. Quantitatively our results and those of [13] agree to within about $10 \%$.

\section{Polarization and geometrical effects}

The importance of both polarization and geometrical effects for the two-photon detachment cross section is clear from Figs. 5 and 7 (which show the frequency interval 1/2 $\lesssim \omega \lesssim 1$ ). Compared with the dependence of the one-photon detachment cross section on both these effects (which enter the result only through the scaled parameter, $\Lambda^{2}$ [4]), for $n$ $=2$ this dependence is much greater. To illustrate this, we present here our analytic result for the two-photon cross section in the approximation $I$ :

$$
\begin{aligned}
\hat{\sigma}_{I}^{(2)}\left(\omega, \mathcal{F}, \Lambda, l, \phi_{1}\right)= & A_{c} \frac{4 \pi^{3} \alpha^{2} \mathcal{F}^{1 / 3}}{15 \omega^{6}}\left\{\left(\mathrm{Ai}^{\prime 2}-\xi A i^{2}\right)\right. \\
& \times\left[32(2 \omega-1)^{2}-l^{2}\left(\omega^{2}+24 \omega-16\right)\right] \\
& +\mathcal{F}^{2 / 3} \mathrm{Ai} \mathrm{Ai}^{\prime}\left[10 l \omega\left(2 l-3 \Lambda^{2} \cos 2 \phi_{1}\right)\right. \\
& \left.+2(2 \omega-1)\left(15 \Lambda^{4}-4 l^{2}-8\right)\right] \\
& +\mathcal{F}^{4 / 3} \mathrm{Ai}^{2}\left[60 \Lambda^{2}\left(1-l \cos 2 \phi_{1}\right)\right. \\
& \left.\left.-7.5 \Lambda^{4}+6 l^{2}+12\right]\right\}
\end{aligned}
$$

One observes that, for an elliptical polarization, with 0 $<|\eta|<1, \hat{\sigma}^{(2)}$ depends on the azimuth angle $\phi$ not only by means of the parameter $\Lambda^{2}=\sin ^{2} \theta(1+l \cos 2 \phi)$, but also by means of the "angle" $\phi_{1}$, where $\tan \phi_{1}=\eta \tan \phi$ [see Eq. (14)]. In Figs. 11 and 12 we extend the results for linear polarization and collinear geometry obtained in Ref. [11] as well as those shown in Fig. 8 to the cases of circular polarization and orthogonal geometry.

Figure 11 shows the frequency dependence of the generalized cross section for two-photon detachment of $\mathrm{H}^{-}$for
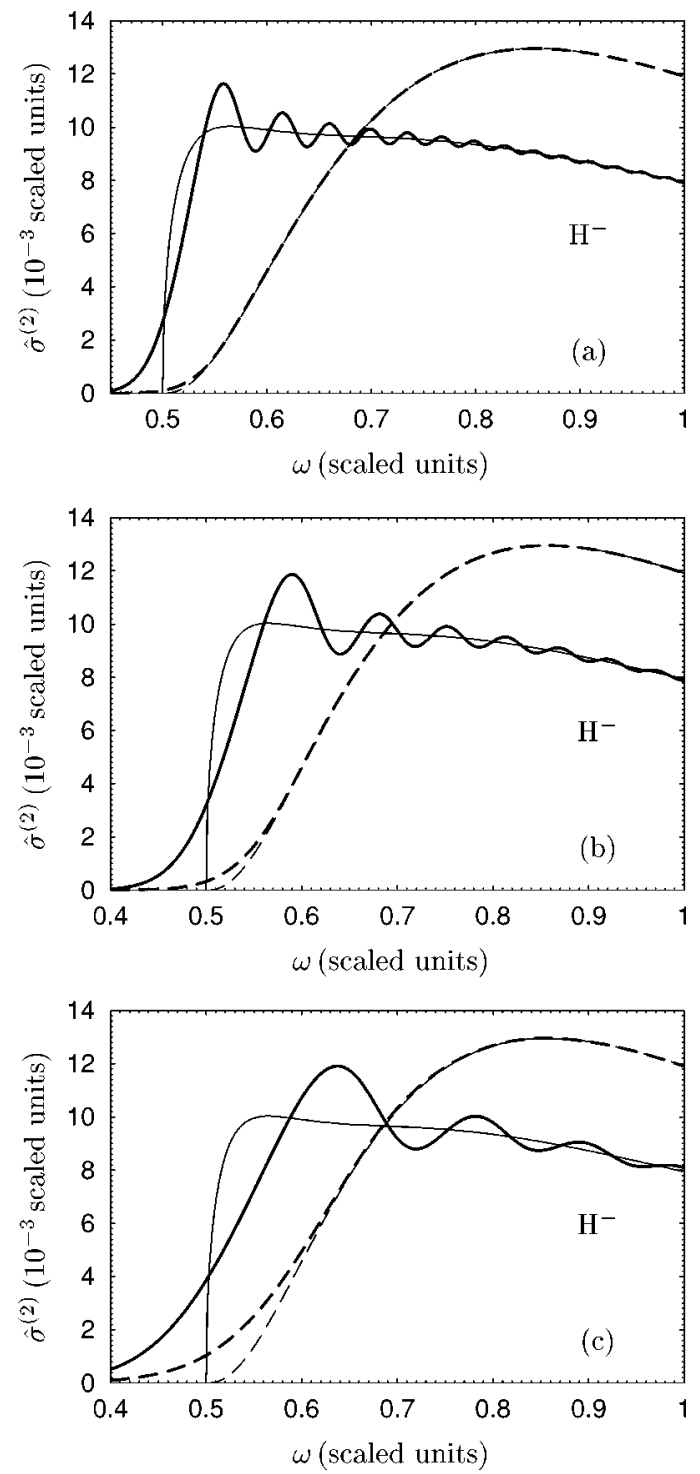

FIG. 11. Frequency dependence of $\hat{\sigma}^{(2)}$ for orthogonal geometries for three values of $\mathcal{F}$ : (a) $\mathcal{F}=0.015$; (b) $\mathcal{F}=0.03$, (c) $\mathcal{F}$ $=0.06$. Thick solid line: linear polarization $(l=1)$ orthogonal to $\mathcal{F}$. Thick dashed line: circular polarization $(l=0)$ in the plane orthogonal to $\mathcal{F}$ (i.e., $\hat{k} \| \mathcal{F}$ ). Thin solid line: linear polarization $(l=1)$ and $\mathcal{F}=0$. Thin dashed line: circular polarization $(l=0)$ and $\mathcal{F}=0$.

orthogonal geometrical arrangements, i.e., in which the laser polarization vector is in the plane orthogonal to the static field $\mathcal{F}$. One observes that for the case of linear polarization and $\mathcal{F} \neq 0$, there is the characteristic oscillation of $\hat{\sigma}^{(2)}$ with increasing frequency. What is surprising is that this occurs for an orthogonal geometry. Such oscillation has not been predicted in one-photon detachment in the orthogonal field configuration. We surmise that it occurs for the two-photon case because the final state includes $s$-wave components, which can be reflected back to the origin by the static field, leading to the observed interference pattern. This interpretation is supported by the absence of such oscillations in Fig. 11 for the case of circular polarization, in which case the final state does not have an $s$-wave component. Figure 11 

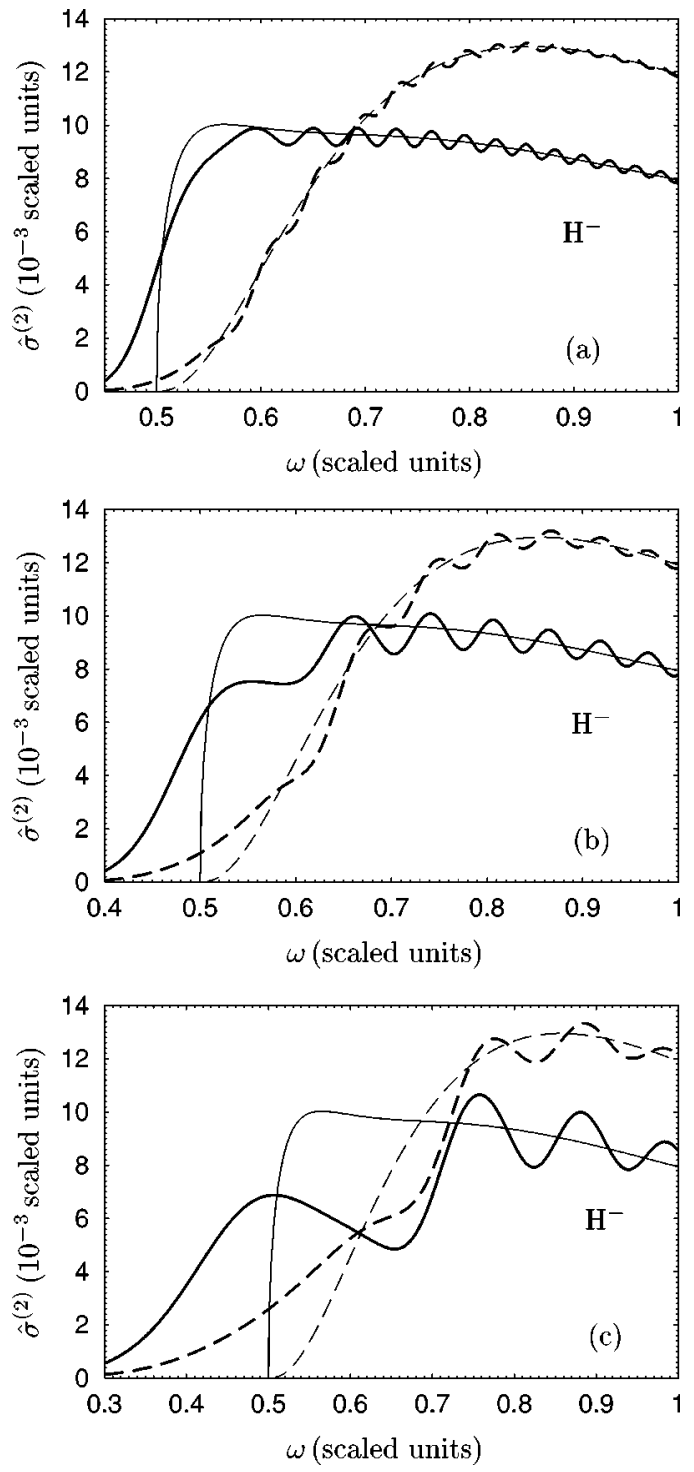

FIG. 12. Frequency dependence of $\hat{\sigma}^{(2)}$ for collinear or coplanar geometries for three values of $\mathcal{F}$ : (a) $\mathcal{F}=0.015$; (b) $\mathcal{F}=0.03$, (c) $\mathcal{F}=0.06$. Thick solid line: linear polarization $(l=1)$ collinear with $\mathcal{F}$. Thick dashed line: circular polarization $(l=0)$ coplanar with $\mathcal{F}$. Thin solid line: linear polarization $(l=1)$ with $\mathcal{F}=0$. Thin dashed line: circular polarization $(l=0)$ with $\mathcal{F}=0$.

shows also results for $\mathcal{F}=0$. One sees that for linear polarization, the result for $\mathcal{F} \neq 0$ oscillates about the one for $\mathcal{F}$ $=0$. For the case of circular polarization the curves for $\mathcal{F}$ $=0$ and $\mathcal{F} \neq 0$ are essentially identical except for the region around the zero-field threshold, i.e., $\omega \approx 0.5$. The difference in this frequency region stems from the effective lowering of the threshold by the static field.

Figure 12 shows, in contrast to Fig. 11, that for geometries in which the laser polarization vector is collinear or coplanar with the static field $\mathcal{F}, \hat{\sigma}^{(2)}$ oscillates as a function of frequency whether the laser polarization is linear or circular. The reason clearly is that in either case the final-state probability amplitude for the electron can be reflected back to the origin by the static field potential, resulting in interfer- ence effects that vary with $\omega$ (or, equivalently, the electron's kinetic energy). For both cases of laser polarization, one sees that the cross section oscillates about its value for $\mathcal{F}=0$ for high enough frequencies above the zero-field threshold, i.e., $\omega>0.5$. However, as $\mathcal{F}$ increases, the oscillations in the region of the zero field threshold $(\omega \approx 0.5)$ are increasingly lower on average for the case of linear polarization than the cross section for $\mathcal{F}=0$. For the case of circular polarization, this lowering occurs after the $\mathcal{F}>0$ and $\mathcal{F}=0$ curves intersect just above the zero-field threshold $(\omega=0.5)$. This effect is due to the lowering of the effective threshold by the static field and the consequent nonzero cross sections for both polarizations below $\omega=0.5$.

\section{Threshold behavior and modified Wigner's law}

As mentioned above and as demonstrated by our results, static-field-induced effects are most important in the threshold domain [i.e., for the conditions opposite to those in the inequality (29)], where $\hat{\sigma}^{(2)}$ vanishes at $\mathcal{F} \rightarrow 0$. We present below the threshold value of $\hat{\sigma}^{(2)}$ for the case of linear polarization and collinear geometry, which follows from Eqs. (28) and (32) for $\omega=1 / 2$ :

$$
\begin{aligned}
\hat{\sigma}_{I}^{(2), t h .}=N \mathcal{F}^{1 / 3}\left[1-\frac{16}{3} \mathcal{F}^{2 / 3} b-\frac{16}{5} \mathcal{F}^{4 / 3} b^{2}\right], \\
\hat{\sigma}_{I I}^{(2), t h .}=N \mathcal{F}^{1 / 3}\left[1-\frac{16}{3} b \mathcal{F}^{2 / 3}\left(1+160 \mathcal{F}^{2}\right)-32 b^{2} \mathcal{F}^{4 / 3}\right. \\
\left.\times\left(\frac{3}{5}+32 \mathcal{F}^{2}\right)-192 \mathcal{F}^{2}+2^{10} \mathcal{F}^{4}\right],
\end{aligned}
$$

where

$$
N=16 \pi \alpha^{2} 3^{1 / 3} \Gamma^{2}(2 / 3) A_{c}, \quad b=-\frac{2 \pi}{3^{5 / 6} \Gamma(2 / 3)^{2}} .
$$

We note the important fact that for two-photon detachment the static-field modification of the threshold behavior is considerably more significant for even weak static fields than in the case of single-photon detachment. In particular, in the weak-field approximation, we have: $\hat{\sigma}^{(2), t h} \sim \mathcal{F}^{1 / 3}$ for twophoton detachment as compared to $\hat{\sigma}^{(1), t h} \sim \mathcal{F}$ for onephoton detachment [4]. Moreover, for $n=2$ the threshold value of $\hat{\sigma}^{(2)}$ depends significantly on the polarization and field geometry, as may be seen from our result for the simplest approximation, $I$ :

$$
\begin{aligned}
\hat{\sigma}_{I}^{(2), t h .}\left(\Lambda, l, \phi_{1}\right)= & N \mathcal{F}^{1 / 3}\left[l^{2}+8 b \mathcal{F}^{2 / 3}\left(\frac{l^{2}}{3}-\frac{l \Lambda^{2} \cos 2 \phi_{1}}{2}\right)\right. \\
& +16 b \mathcal{F}^{4 / 3}\left(\frac{2+l^{2}}{10}\right. \\
& \left.\left.+\Lambda^{2}\left(1-l \cos 2 \phi_{1}\right)-\frac{\Lambda^{4}}{8}\right)\right] .
\end{aligned}
$$




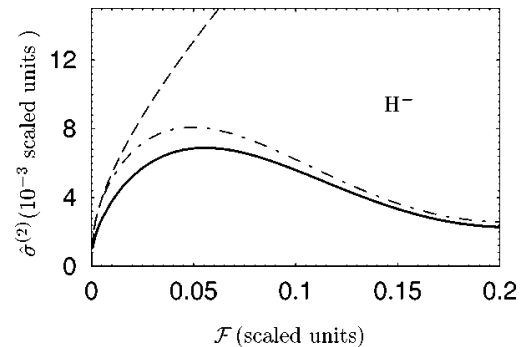

FIG. 13. Threshold behavior of $\hat{\sigma}^{(2)}(\mathcal{F})$ [i.e., for $\omega=1 / 2$ (in scaled units)] for linear polarization $(l=1)$ and the collinear field geometry as a function of static field amplitude, $\mathcal{F}$. Solid line: exact result [from Eqs. (17) and (26)]. Dashed line: approximation I [from Eq. (34)]. Dot-dashed line: approximation $I I$ [from Eq. (35)].

Thus, whereas for the case of linear polarization (when the $s$-wave continuum channel contributes to the cross section) we find that $\hat{\sigma}_{I}^{(2), t h .} \sim \mathcal{F}^{1 / 3}$, for the case of circular polarization (when only the $d$-wave channel contributes) the field dependence of $\hat{\sigma}^{(2), t h}$ is much more suppressed $\left(\sim \mathcal{F}^{5 / 3}\right)$. These results illustrate how a static electric field modifies significantly the well-known Wigner threshold laws [24] for ionization of a particle bound in a short-range potential. Instead of the usual low-energy dependence $\sigma_{L}(E) \sim E^{L+1 / 2}$ of the partial cross sections (or the equivalent for the scattering phases) which correspond to the angular momentum $L$ of a weakly bound electron in the continuum (with energy $E$ $=n \omega-1$ for $n$-photon detachment), in the presence of a (weak) static field the cross sections are finite at $E=0$. This well-known qualitative fact follows from one-electron considerations, has been observed experimentally [28], and has been confirmed by many-electron numerical calculations $[12,13]$. Our analytical results for an arbitrary geometry and laser polarization allow one to formulate the modification of Wigner laws in a weak static electric field quantitatively. Namely, the threshold behaviors of the cross sections for small energies $\left(E \ll \mathcal{F}^{2 / 3}\right)$ of the escaping electron are determined by the characteristic field parameter, $\mathcal{F}^{2 / 3}$,

$$
\sigma(E=0) \sim\left(\mathcal{F}^{2 / 3}\right)^{L+1 / 2} .
$$

Obviously, if the final continuum state of the escaping electron is a superposition of states with different angular momenta $L$, only the minimal one enters the modified Wigner law in Eq. (37).

Figure 13 demonstrates the threshold behavior of $\hat{\sigma}^{(2)}(\mathcal{F})$ obtained from exact results for $\operatorname{Im} \gamma(\mathcal{F} ; \omega=1 / 2)$ and its comparison with results for approximations $I$ and $I I$. One observes that $\hat{\sigma}_{I I}^{(2), t h}$ is in reasonable agreement with the exact result. However, the approximation $I$ is reasonable only for weak static fields $\mathcal{F}$ and is qualitatively wrong for strong static fields $\mathcal{F}$.

Figure 14 demonstrates the threshold behavior of the $\sim F^{4}$ contribution $\Gamma^{(4)}$ to the total detachment rate in Eq. (25), extracted from the exact results for $\operatorname{Im} \gamma(\mathcal{F} ; \omega=1 / 2)$ for different laser polarizations and field geometries. One observes in all cases that $\Gamma^{(4)}$ increases from its zero static field $(\mathcal{F}$ $=0$ ) value as $\mathcal{F}$ increases, reaches a maximum in the vicinity
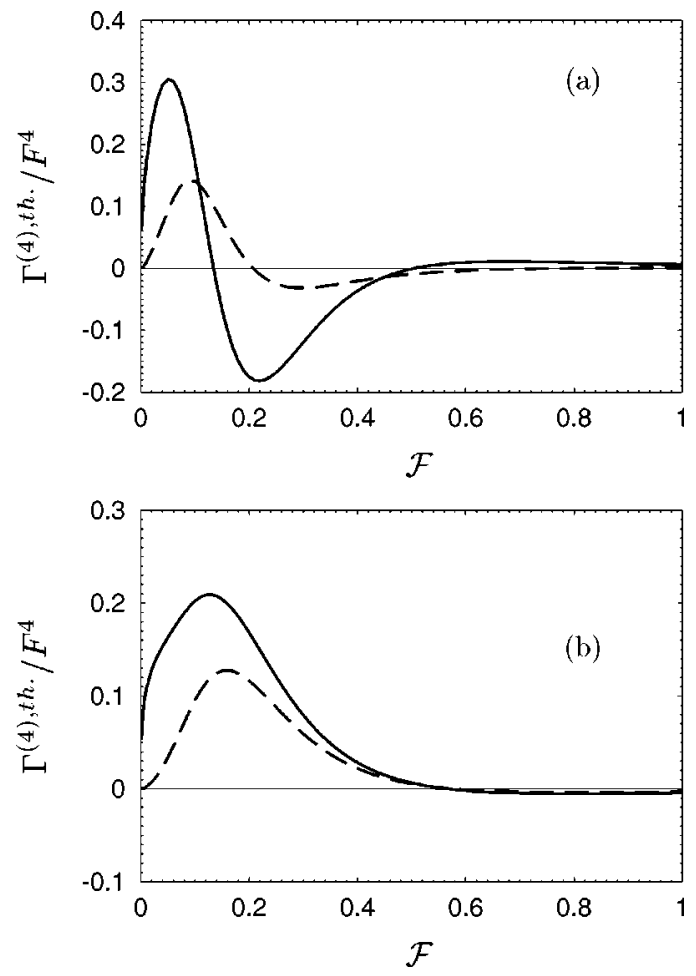

FIG. 14. Threshold behavior of $\operatorname{Im} \gamma(\mathcal{F} ; \omega=1 / 2) / 12=\Gamma^{(4)} / F^{4}$ for: (a) orthogonal field geometry $(l=0,1)$; (b) collinear $(l=1)$ or coplanar $(l=0)$ field geometry. Solid line: linearly polarized $(l$ $=1)$ laser field. Dashed line: circularly polarized $(l=0)$ laser field. Scaled units are used.

of $\mathcal{F} \approx 0.1$, and then decreases, becoming approximately zero (on the scale shown) for $\mathcal{F}>0.5$. However, whereas for the collinear $(l=1)$ and coplanar $(l=0)$ field geometries this decrease is essentially monotonic in the range $0.2 \leq \mathcal{F} \leq 0.5$, for the orthogonal field geometries $\Gamma^{(4)}$ becomes negative in this intermediate range of $\mathcal{F}$, indicating that this higher-order contribution reduces the overall decay rate $\Gamma$ in Eq. (25). Finally, one observes from Fig. 14 that the variation of the threshold value of $\Gamma^{(4)}$ with $\mathcal{F}$ is less pronounced for a circularly polarized $(l=0)$ laser field than for a linearly polarized $(l=1)$ laser field regardless of the field geometry.

\section{B. Results for alternative laser polarizations and field geometries for $\omega>1$}

An interesting qualitative result on the frequency behavior of $\gamma(\mathcal{F} ; \omega)$ is evident from Figs. 4-7. Namely, although both $\operatorname{Re} \gamma$ and $\operatorname{Im} \gamma$ oscillate for $\omega \gtrsim 1 / 2$, their oscillation patterns (i.e., periods, amplitudes, and signs of $\operatorname{Re} \gamma$ and $\operatorname{Im} \gamma$ ) are very different in the regions below and above the singlephoton, zero-field threshold, $\omega<1$ and $\omega>1$. Physically, these differences originate from the different physical phenomena that are described by $\operatorname{Im} \gamma(\mathcal{F} ; \omega)$ for the two frequency intervals. [Obviously, $\operatorname{Re} \gamma$, which gives the linear in laser intensity correction to the Stark shift, repeats the peculiarities of the $\omega$ dependence of $\operatorname{Im} \gamma$ because they are the real and the imaginary part of the same analytical function, $\epsilon=\epsilon(\omega)$.] For $\omega<1$, two-photon detachment is the only 
channel that allows laser-induced decay of the quasistationary (because of the static field) state $\Phi_{E}(\mathbf{r})$. For $\omega>1$, the one-photon channel is open; therefore for this case the term $\Gamma^{(2)}=(1 / 2) \operatorname{Im} \alpha(\mathcal{F} ; \omega) F^{2}$ in Eq. (25) is dominant. Generally, for $\omega>1$ the next order in $F^{2}$ correction, $\Gamma^{(4)}$ (i.e., $\operatorname{Im} \gamma$ ), describes both above-threshold, two-photon detachment (with a rate, say, $\Gamma_{a t d}^{(4)}$ ) and the linear in laser intensity correction (say, $\Gamma_{I}^{(4)}$ ) to the one-photon rate, $\Gamma^{(2)}$. However, for moderate $\mathcal{F}$, it is impossible to present our exact analytical result for $\Gamma^{(4)}$ simply as the sum of $\Gamma_{I}^{(4)}$ and $\Gamma_{\text {atd }}^{(4)}$ since such a separation is impossible because of the interference between these two channels. Nevertheless, the results in Figs. 5 and 7 allow one to obtain some important information on the relative magnitudes of the terms discussed. Obviously, the (total) rate for two-photon detachment is a manifestly positive observable for the whole interval of frequencies $\omega$ $>1 / 2$. Indeed, for frequencies below the one-photon threshold, where the one-photon detachment rate $\Gamma^{(2)}$ vanishes, $\Gamma^{(4)}$ describes two-photon detachment only (neglecting the exponentially small effects of static field ionization and stimulated emission) and it is positive, as it should be. On the other hand, for $\omega>1$ the situation is different: $\Gamma^{(4)}$ is negative for orthogonal field geometry (Fig. 5) or its sign oscillates with increasing $\omega$ (Fig. 7). These results imply that, in the above-threshold region, the nonlinear in laser intensity corrections to the (linear in intensity) perturbative result for the photodetachment rate $\Gamma^{(2)}$ are more important for $\Gamma^{(4)}$ quantitatively than the direct two-photon detachment contribution to $\Gamma^{(4)}$. Moreover, the (mainly) negative sign of the nonlinear corrections indicates that, as the intensity $I$ increases, the slope of the total decay rate, $\Gamma(I)$, has a tendency to decrease. Such behavior is similar to the onset of a stabilizationlike behavior of the photodetachment rate for a weakly bound level in a strong laser field for $\mathcal{F}=0$ [29]. On the other hand, for some intervals of $\omega$ the sign of $\Gamma^{(4)}$ may be positive, and thus, one observes also $d \Gamma(I) / d I>0$. Therefore, a moderate static field may provide significant control of the photodetachment decay rate in a strong laser field.

The frequency dependence of $\Gamma^{(4)}$ in the above-threshold domain is clear from Figs. 5 and 7. To illustrate the $\mathcal{F}$ and $F$ dependencies of the total laser-induced rate, $\Gamma_{\text {las. }}=\Gamma^{(2)}$ $+\Gamma^{(4)}$, we present results for the frequency $\omega_{1}=1.5468$ that has been used in the recent experiment [30] for measuring the angular distribution in two-photon detachment of $\mathrm{H}^{-}$by a linearly polarized laser field. [Note that the total cross sections for $\omega=\omega_{1}$ measured in the experiment [30], $\hat{\sigma}^{(1)}$ $=(3.6 \pm 1.7) \times 10^{-17} \mathrm{~cm}^{2}$ and $\hat{\sigma}^{(2)}=(1.3 \pm 0.5) \times 10^{-48} \mathrm{~cm}^{4}$ $\mathrm{sec}$, are in good agreement with our calculated results for the zero-range potential model (for $\mathcal{F}=0$ ), $\sigma^{(1)}=3.648$ $\times 10^{-17} \mathrm{~cm}^{2}$ and $\sigma^{(2)}=0.962 \times 10^{-48} \mathrm{~cm}^{4} \mathrm{sec}$.] In Fig. 15 we present the $\mathcal{F}$ dependence of $\Gamma_{\text {las. }}$ for a fixed value of $F$ and for different field geometries and laser polarizations. Similarly, in Fig. 16 we present the laser amplitude $F$ dependence of $\Gamma_{\text {las. }}$ up to values for $F$ at which $\Gamma^{(2)}$ and $\Gamma^{(4)}$ become comparable.

For a linearly polarized laser field in the collinear geometry [indicated by the thick solid line in Fig. 15(b)], the static-field-dependent oscillatory structure of the photode-
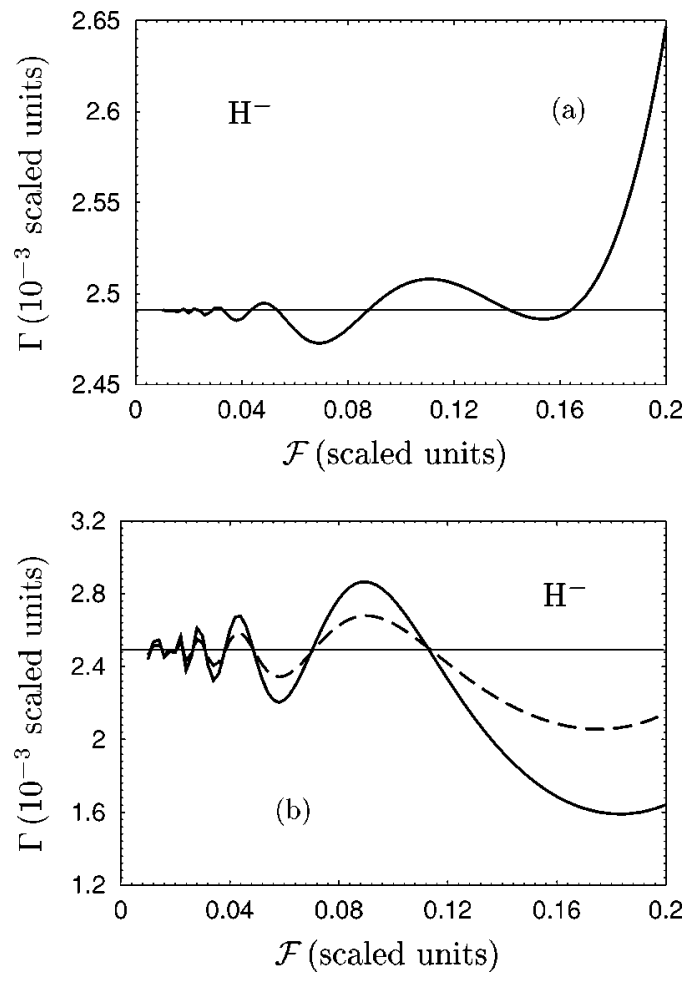

FIG. 15. $\mathcal{F}$ dependence of the total detachment rate, $\Gamma$ [cf. Eq. (25)], at $\omega=1.5468$ and $F=0.1$ for (a) orthogonal and (b) collinear $(l=1)$ or coplanar $(l=0)$ field geometries. Thick solid line: $l=1$. Thick dashed line: $l=0$. Thin line: result for $\mathcal{F}=0$. Note that for orthogonal field geometries and for the case $\mathcal{F}=0$, differences between the results for linearly $(l=1)$ and circularly $(l=0)$ polarized light are not observable on the scale of this figure.

tachment rates is well known from earlier (lowest order in the laser field) model calculations (as discussed in Ref. [4]) and has been confirmed in Refs. $[12,13]$ by accurate nonperturbative numerical analyses that take into account electron correlation effects. One sees in Fig. 15 that regardless of the geometry or the type of laser polarization, the rate $\Gamma_{\text {las. }}$ oscillates about its $\mathcal{F}=0$ value until, at sufficiently large values of $\mathcal{F}$, static field detachment becomes dominant. One observes also that these oscillations are of much smaller amplitude for orthogonal field configurations than for collinear $(l$ $=1)$ or coplanar $(l=0)$ field configurations. In Fig. 16 we see that in general as the laser amplitude $F$ increases, $\Gamma_{\text {las }}$. tends to reach a maximum and then decreases. However, for the orthogonal field configurations these maxima lie within a narrow range, whereas for the collinear $(l=1)$ and coplanar $(l=0)$ field configurations the maxima take much lower values for the highest static field values shown; also, for the lowest nonzero static field value, the maxima are higher than for the $\mathcal{F}=0$ maximum.

Our numerical results for the polarizability and hyperpolarizability allow an estimation of the critical amplitude of the laser field, at which the terms $\sim F^{2}$ and $\sim F^{4}$ on the right-hand side (rhs) of Eq. (15) have comparable amplitude, thereby implying that perturbation theory in $F$ for the Stark shift and width (i.e., the decay rate) breaks down. This critical field is determined by the relation between $\alpha(\mathcal{F} ; \omega)$ and 

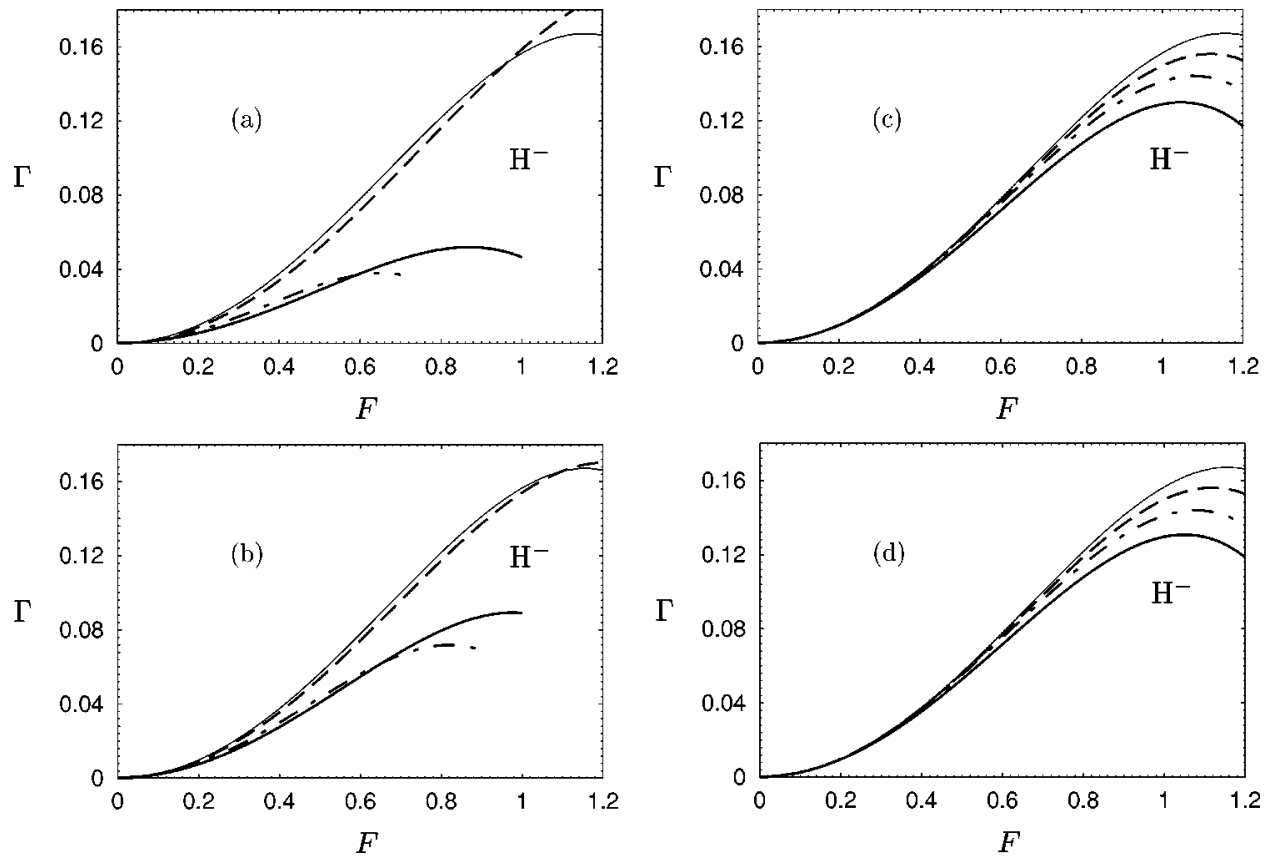

FIG. 16. $F$ dependence of the total detachment rate [cf. Eq. (25)], $\Gamma$, at $\omega=1.5468$ for a number values of the static field strength $\mathcal{F}$ for (a) collinear ( $l$ $=1$ ) geometry; (b) coplanar ( $l$ $=0$ ) geometry; (c) orthogonal ( $l$ =1) geometry; and (d) orthogonal $(l=0)$ geometry. Thick solid line: $\mathcal{F}=0.2$. Thick dot-dashed line: $\mathcal{F}=0.15$. Thick dashed line: $\mathcal{F}=0.06$. Thin solid line: $\mathcal{F}=0$. Scaled units are used.
$\gamma(\mathcal{F} ; \omega)$, and, obviously, it depends significantly on the field geometry and the polarization state of $\mathbf{F}(t)$. Moreover, it may be different for the real and imaginary parts of the quasienergy. As an example, in Fig. 17 we present the frequency dependence of the laser field amplitude $F_{c r}$. for the case of a linearly polarized laser field $(l=1)$ in the collinear field geometry for the situation in which the modulus of the second term on the rhs of Eq. (15) equals $10 \%$ of the modulus of the first term on the rhs of Eq. (15), i.e.,

$$
F_{c r .}=\sqrt{0.6 \frac{|\alpha(\mathcal{F} ; \omega)|}{|\gamma(\mathcal{F} ; \omega)|}}
$$

One sees from Fig. 17 that perturbative estimations of the decay rate are possible up to $F \leqq 0.2$. Also, this critical field increases monotonically with increasing $\omega$.

\section{CONCLUSIONS}

We have performed an analysis of second order in laser intensity effects in the interaction of a weakly bound electron

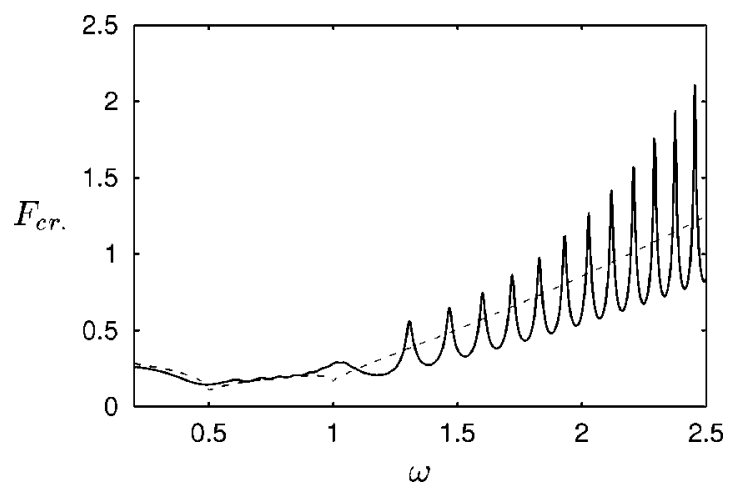

FIG. 17. Frequency dependence of the critical laser field amplitude, $F_{c r}$. in Eq. (38) for the case of a linearly polarized $(l=1)$ laser field and a collinear field geometry $(\Lambda=\sqrt{2})$. Solid line: $\mathcal{F}$ $=0.03$. Dotted line: $\mathcal{F}=0$. Scaled units are used. (e.g., as in $\mathrm{H}^{-}$) with both laser and static electric fields. The laser field effects are taken into account perturbatively assuming that, for frequencies comparable with the electron binding energy, the two lowest orders of perturbation theory suffice to give reasonable results up to moderate values of the laser field amplitude. On the other hand, it is well known that static-field ionization effects cannot be analyzed perturbatively in the strength of the static field. Thus, in our formulation these effects are taken into account exactly using quasistationary (or resonance) states having the proper outgoing wave asymptotics for an electron in a static field. We discussed generally the atomic response to a monochromatic laser field in terms of the dynamic hyperpolarizability tensor generalized for the case of a decaying [in strong external field(s)] quantum system. This generalization was performed using the complex quasienergy approach with properly normalized quasistationary wave functions [4]. After establishing the general structure of the hyperpolarizability tensor (using general symmetry arguments) for an atomic system in a strong static electric field in terms of five irreducible components, we performed analytical calculations of these components for the zero-range potential model in terms of Airy functions, in a way similar to those used to obtain the dynamic polarizability tensor for this model potential in Ref. [4].

Our analytical results and numerical evaluations for higher-order effects in the laser intensity demonstrate features of the interaction of a bound electron with photons in the presence of a static electric field that are quite different from those found in our analysis of the linear in intensity case [4]. First, they show that the typical oscillation pattern in the frequency dependence of both real and imaginary parts of the nonlinear susceptibilities exists for any geometry of the static and laser fields, including the case of orthogonal geometry (when the vector $\mathcal{F}$ is orthogonal to the plane of the polarization ellipse of a laser field). Moreover, this oscillation pattern is extremely sensitive not only to the geometry, 
but also to the polarization state of $\mathbf{F}(t)$. Second, qualitatively we found that both the periods and the amplitudes of oscillations are rather different for the frequency intervals $\omega>1$ and $\omega<1$. These differences stem from the different physical processes that are described by the imaginary part of the hyperpolarizability in these two frequency intervals: these are, respectively, the direct two-photon detachment (for $\omega<1)$ and the stimulated re-emission of a photon in singlephoton detachment (for $\omega>1$ ). This second channel (but not above-threshold, two-photon detachment) gives the dominant correction to the total photodetachment rate for not too strong laser fields (i.e., for fields that may be treated perturbatively).

Finally, our general results for an arbitrary field geometry and laser polarization have allowed a detailed analysis of the threshold behavior of the hyperpolarizability. Our analyses demonstrate that the cross sections for photoprocesses are finite at thresholds and for weak $\mathcal{F}$ they are determined by the characteristic parameter $\mathcal{F}^{2 / 3}$ and by the minimal value of the angular momentum $L$ of the detached electron. Thus, these results present the static-electric-field-induced modification of Wigner's law for the threshold behavior of cross sections of photoprocesses from a weakly bound system.

\section{ACKNOWLEDGMENTS}

The authors thank I. I. Fabrikant for useful discussions. This work was supported in part by RFBR Grant No. 00-0217843, by INTAS-RFBR Grant No. 97-693, by Russian Ministry of Education Grant No. E00-3.2-515 (M.V.F. and N.L.M.), and by NSF Grant No. PHY-0070980.
[1] Yu. N. Demkov and V. N. Ostrovsky, Zero-Range Potentials and Their Applications in Atomic Physics (New York, Plenum, 1988).

[2] N. L. Manakov and A. G. Fainshtein, Zh. Exp. Teor. Fiz. 79, 751 (1980) [Sov. Phys. JETP 52, 382 (1980)].

[3] W. Becker, S. Long, and J. K. McIver, Phys. Rev. A 50, 1540 (1994).

[4] N. L. Manakov, M. V. Frolov, A. F. Starace, and I. I. Fabrikant, J. Phys. B 33, R141 (2000).

[5] I. N. Arutyunyan and G. A. Askar'yan, Pis'ma Zh. Éksp. Teor. Fiz. 12, 378 (1970) [JETP Lett. 12, 259 (1970)].

[6] A. I. Nikishov, Zh. Exp. Teor. Fiz. 62, 562 (1972) [Sov. Phys. JETP 35, 298 (1972)].

[7] V. Z. Slonim and F. I. Dalidchik, Zh. Exp. Teor. Fiz. 71, 2057 (1976) [Sov. Phys. JETP 44, 1081 (1976)]

[8] N. B. Delone, N. L. Manakov, and A. G. Fainshtein, Zh. Exp. Teor. Fiz. 86, 906 (1984) [ Sov. Phys. JETP 59, 529 (1984)].

[9] V. N. Ostrovsky and D. A. Telnov, J. Phys. B 23, L477 (1991); 26, 415 (1993); Laser Phys. 3, 495 (1993).

[10] B. Gao and A. F. Starace, Phys. Rev. A 42, 5580 (1990).

[11] M. Q. Bao, I. I. Fabrikant, and A. F. Starace, Phys. Rev. A 58, 411 (1998).

[12] C. A. Nicolaides and Th. Mercouris, Chem. Phys. Lett. 159, 45 (1989).

[13] Th. Mercouris and C. A. Nicolaides, J. Phys. B 23, 2037 (1990); Note that according to Ref. [6] in the reference list of C. Harritos, T. Mercouris, and C. A. Nicolaides, Phys. Rev. A 63, 013410 (2001), there is an error in this 1990 paper. Namely, the values of the intensities in the figures must be reduced by a factor two.

[14] Th. Mercouris and C. A. Nicolaides, J. Phys. B 24, L57 (1991).

[15] N. L. Manakov, M. A. Preobrazhenskii, L. P. Rapoport, and A. G. Fainshtein, Zh. Éksp. Teor. Fiz. 75, 1243 (1978) [Sov. Phys. JETP 48, 626 (1978)].

[16] C. A. Nicolaides, Th. Mercouris, and N. A. Piangos, J. Phys. B 23, L669 (1990).

[17] J. Pipin and D. M. Bishop, J. Phys. B 25, 17 (1992).

[18] The necessity for the introduction of the dual functions, $\widetilde{\Phi}_{\epsilon}$, stems from the fact that for decaying states the quasienergy $\epsilon$ is complex. This implies that the Hamiltonian for the QQES is not Hermitian, and thus, the QQES are not normalizable in the usual way. Just as for resonance states in time-independent problems, one needs to introduce a dual set of states with which to calculate normalization integrals and matrix elements [e.g., such as $\widetilde{\mathbf{d}}_{ \pm \omega}$ in Eq. (6)]. These issues have been discussed in detail (for the current problem of laser detachment of an electron from a short-range potential in the presence of a strong static field), and numerous references have been given, in a recent review [4].

[19] A. G. Fainshtein, N. L. Manakov, V. D. Ovsiannikov, and L. P. Rapoport, Phys. Rep. 210, 111 (1992).

[20] M. L. Du and J. B. Delos, Phys. Rev. A 38, 5609 (1988); see also p. R157 of Ref. [4].

[21] N. L. Manakov, V. D. Ovsiannikov, and L. P. Rapoport, Phys. Rep. 141, 319 (1986).

[22] Handbook of Mathematical Functions, edited by $\mathrm{M}$. Abramowitz and I. A. Stegun (Dover, New York, 1965), Sec. 10.4 .

[23] M. V. Frolov, Ph.D. thesis, Voronezh State University, 2000 (unpublished).

[24] E. P. Wigner, Phys. Rev. 73, 1002 (1948); A. I. Baz', Ya. B. Zel'dovich, and A. M. Perelomov, Scattering, Reactions and Decays in Nonrelativistic Quantum Mechanics, 2nd ed. (Nauka, Moscow, 1971).

[25] S. A. Adelman, Phys. Rev. A 5, 508 (1972).

[26] C. Rangan and A. R. P. Rau, Phys. Rev. A 61, 033405 (2000).

[27] I. I. Fabrikant, M. V. Frolov, N. L. Manakov, and A. F. Starace, Phys. Rev. A (to be published).

[28] H. C. Bryant, V. Yuan, C. R. Hummer, W. W. Smith, S. Cohen, W. P. Reinhardt, and L. Overman, Phys. Rev. Lett. 58, 2412 (1987).

[29] N. L. Manakov, M. V. Frolov, B. Borca, and A. F. Starace, Pis'ma Zh. Éksp. Teor. Fiz. 72, 426 (2000) [JETP Lett. 72, 294 (2000)].

[30] X. M. Zhao, M. S. Gulley, H. C. Bryant, C. E. M. Strauss, D. J. Funk, A. Stintz, D. C. Rislove, G. A. Kyrala, W. B. Ingalls, and W. A. Miller, Phys. Rev. Lett. 78, 1656 (1997); 60, 4753 (1999). 